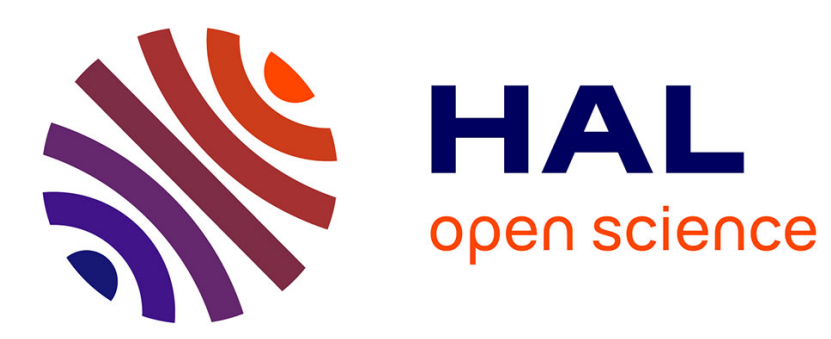

\title{
Tabu search for the cyclic bandwidth problem
}

Eduardo Rodriguez-Tello, Hillel Romero-Monsivais, Gabriel Ramirez-Torres, Frédéric Lardeux

\section{To cite this version:}

Eduardo Rodriguez-Tello, Hillel Romero-Monsivais, Gabriel Ramirez-Torres, Frédéric Lardeux. Tabu search for the cyclic bandwidth problem. Computers and Operations Research, 2015, 57, pp.17-32. 10.1016/j.cor.2014.11.013 . hal-01392218

\section{HAL Id: hal-01392218 \\ https://hal.science/hal-01392218}

Submitted on 4 Nov 2021

HAL is a multi-disciplinary open access archive for the deposit and dissemination of scientific research documents, whether they are published or not. The documents may come from teaching and research institutions in France or abroad, or from public or private research centers.
L'archive ouverte pluridisciplinaire HAL, est destinée au dépôt et à la diffusion de documents scientifiques de niveau recherche, publiés ou non, émanant des établissements d'enseignement et de recherche français ou étrangers, des laboratoires publics ou privés. 


\title{
Tabu Search for the Cyclic Bandwidth Problem ${ }^{\text {th }}$
}

\author{
Eduardo Rodriguez-Tello,a, Hillel Romero-Monsivais ${ }^{\mathrm{a}}$, Gabriel Ramirez-Torres ${ }^{\mathrm{a}}$, Frédéric Lardeux ${ }^{\mathrm{b}}$ \\ ${ }^{a}$ CINVESTAV-Tamaulipas, Information Technology Laboratory \\ Km. 5.5 Carretera Victoria-Soto La Marina, 87130 Victoria Tamps., Mexico \\ ${ }^{b}$ LERIA, Université d'Angers. \\ 2 Boulevard Lavoisier, 49045 Angers, France
}

\begin{abstract}
The Cyclic Bandwidth problem (CB) for graphs consists in labeling the vertices of a guest graph $G$ by distinct vertices of a host cycle $C_{n}$ (both of order $n$ ) in such a way that the maximum distance in the cycle between adjacent vertices in $G$ is minimized. To the best of our knowledge, this is the first research work investigating the use of metaheuristic algorithms for solving this challenging combinatorial optimization problem in the case of general graphs.

In this paper a new carefully devised Tabu Search algorithm, called TScB, for finding near-optimal solutions for the CB problem is proposed. Different possibilities for its key components and input parameter values were carefully analyzed and tuned, in order to find the combination of them offering the best quality solutions to the problem at a reasonable computational effort.

Extensive experimentation was carried out, using 113 standard benchmark instances, for assessing its performance with respect to a Simulated Annealing ( $\mathrm{SAcB}$ ) implementation. The experimental results show that there exists a statistically significant performance amelioration achieved by TSсв with respect to SAсв in 90 out of 113 graphs (79.646\%). It was also found that our TScв algorithm attains 56 optimal solutions and establishes new better upper bounds for the other 57 instances. Furthermore, these competitive results were obtained expending reasonable computational times.
\end{abstract}

Key words:

cyclic bandwidth problem, tabu search, best-known bounds

\section{Introduction}

The Cyclic Bandwidth problem (CB) is a graph embedding problem. It was first stated by Leung et al. in 1984 in relation with the design of a ring interconnection network [1]. Their aim was to find an arrangement on a cycle for a set $V$ of computers with a known communication pattern, given by the graph $G(V, E)$, in such a way that every message sent can arrive at its destination in at most $k$ steps. The decision problem corresponding to the CB is known to be NPcomplete [2], and arises also in other important application areas like VLSI designs [3], data structure representations [4], code design [5] and interconnection networks for parallel computer systems [6].

The CB problem can be formally defined as follows. Let $G(V, E)$ be a finite undirected graph (guest) of order $n$ and $C_{n}$ a cycle graph (host) with vertex set $\left|V^{\prime}\right|=n$ and edge set $E^{\prime}$. Given an injection $\varphi: V \rightarrow V^{\prime}$, which represents an embedding of $G$ in $C_{n}$, the cyclic bandwidth (the cost) for $G$ with respect to $\varphi$ is defined as:

$$
B_{C}(G, \varphi)=\max _{u v \in E}\left\{|\varphi(u)-\varphi(v)|_{n}\right\}
$$

where $|x|_{n}=\min \{|x|, n-|x|\}(1<|x|<n-1)$ is called the cyclic distance, and $\varphi(u)$ denotes the label associated to vertex $u$.

\footnotetext{
This research work was partially funded by the following projects: 51623 Fondo Mixto CONACyT y Gobierno del Estado de Tamaulipas; CONACyT 99276, Algoritmos para la Canonización de Covering Arrays.

${ }^{*}$ Corresponding author.

Email addresses: ertello@tamps. cinvestav.mx (Eduardo Rodriguez-Tello), hromero@tamps.cinvestav .mx (Hillel Romero-Monsivais), grtorres@tamps . cinvestav .mx (Gabriel Ramirez-Torres), lardeux@info.univ-angers .fr (Frédéric Lardeux) 


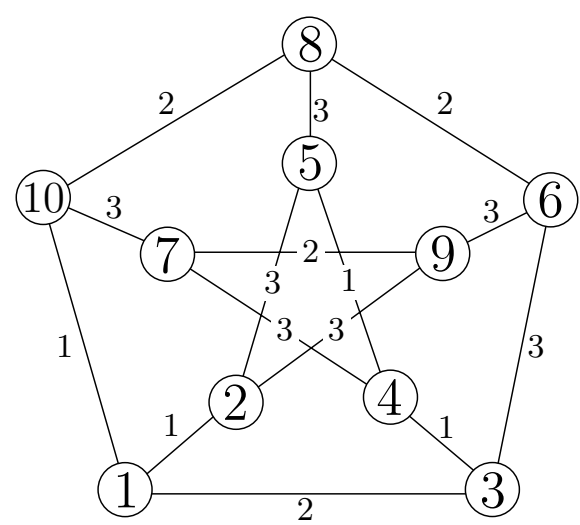

(a) A labeling $\varphi$ for graph $G$.

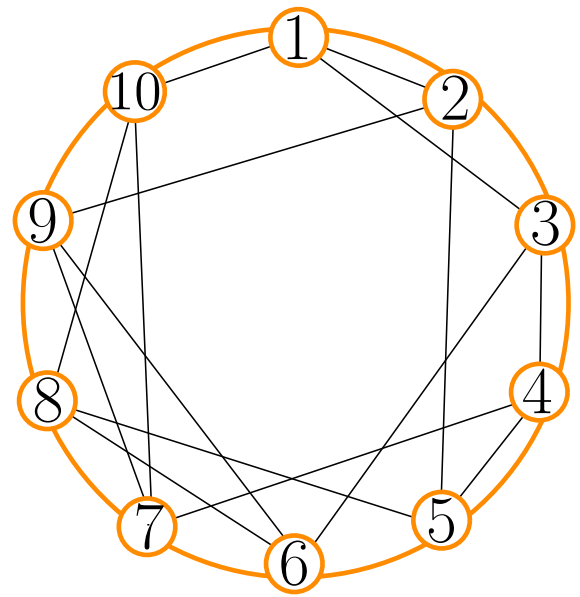

(b) $G$ embedded in the cycle $C_{n}$

Figure 1: Example of a cyclic bandwidth problem instance.

Then the CB problem consists in finding an embedding $\varphi^{*}$, such $B_{C}\left(G, \varphi^{*}\right)$ is minimum, i.e.,

$$
B_{C}\left(G, \varphi^{*}\right)=\min _{\varphi \in \mathscr{E}}\left\{B_{C}(G, \varphi)\right\}
$$

where $\mathscr{E}$ is the set of all possible embeddings. The embedding $\varphi^{*}$ satisfying this condition is called an optimal embedding.

Note that an embedding can also be seen as a labeling of the guest graph $G$ using distinct vertices of the host graph $C_{n}{ }^{1}$. The cost of such an embedding is the maximum distance in $C_{n}$ between two adjacent vertices in the guest graph $G$.

For instance, consider the graph $G(V, E)$ of order $n=10$ depicted in Figure 1(a) with the labeling $\varphi$ given by the numbers shown inside each vertex. The cyclic distance of each edge $u v \in E$ is calculated using the expression $\min \{|\varphi(u)-\varphi(v)|, n-|\varphi(u)-\varphi(v)|\}$ and represented by the number associated to that edge. For this particular labeling $\varphi$, the cyclic bandwidth of $G$ is $B_{C}(G, \varphi)=3$. The resulting embedding of the graph $G$ in a cycle graph $C_{n}$ is presented in Figure 1(b) for illustrative purposes.

The CB problem is a natural extension of the well-known bandwidth minimization (BM) problem for graphs [7], which consists in embedding the vertices of a guest graph $G$ in a host path $P$ (both of order $n$ ) in such a way that the maximum distance in the path between adjacent vertices in $G$ is minimized. Formally, the bandwidth (the cost) for $G$ with respect to $\varphi$ is defined as:

$$
B_{P}(G, \varphi)=\max _{u v \in E}\{|\varphi(u)-\varphi(v)|\} .
$$

There exist some special graphs (2- and 3-dimensional meshes, hypercubes, complete trees) for which it has been demonstrated that their optimal cyclic bandwidth and bandwidth are equal $[6,8]$. It has permitted to establish the following bound relation between bandwidth $B_{P}(G)$ and cyclic bandwidth $B_{C}(G)$ of a general graph [6]:

$$
\frac{1}{2} B_{P}(G) \leq B_{C}(G) \leq B_{P}(G)
$$

Even if both combinatorial optimization problems are related, an algorithm specially devised for one of them is not expected to perform well on the other. This is illustrated by considering as example the graph $i b m 32$ from the Harwell-Boeing Sparse Matrix Collection ${ }^{2}$ (other tested graphs give similar results), which is composed of $n=32$

\footnotetext{
${ }^{1}$ Hereafter the terms embedding and labeling are used indistinctly.

${ }^{2}$ http://math.nist.gov/MatrixMarket/data/Harwell-Boeing
} 


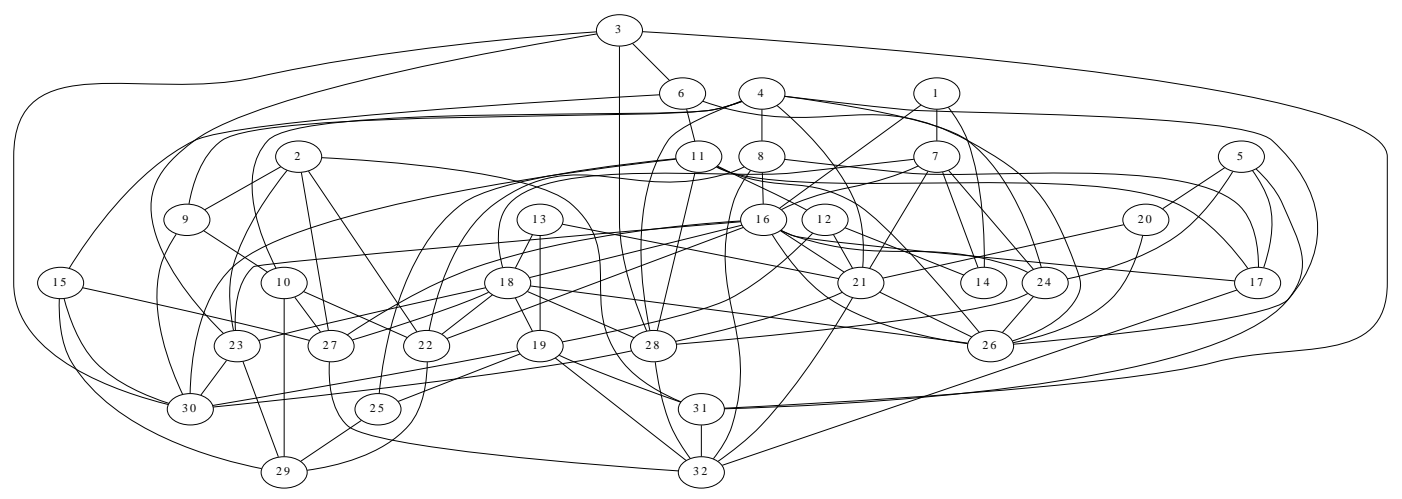

Figure 2: Graph ibm32 from the Harwell-Boeing Sparse Matrix Collection.

vertices (see Figure 2). For this particular graph 1000 local optimal embeddings were generated by executing a first improvement descent algorithm for the CB problem, starting from different randomly generated initial solutions (embeddings). Then, for each of the resulting embeddings the cyclic bandwidth and the bandwidth objective functions were computed according to (1) and (3), respectively. The Pearson's correlation coefficient between these two sets of values is small ( $r=-0.18$ ), showing the weak correlation (if any) among the solutions of both problems. Furthermore, the embedding with the optimal cyclic bandwidth $\left(B_{C}(G)=9\right)$ for this particular graph results in bandwidth value of 31 , which is very far from the optimal value $\left(B_{P}(G)=11\right)$ [9]. In this context there exist a real need for devising new algorithms specially devoted for solving the $\mathrm{CB}$ problem.

This paper aims at developing, to the extent of our knowledge, the first tabu search (TS) algorithm implementation (hereafter called $\mathrm{TS} \mathrm{CB}$ ) for finding near-optimal solutions for the $\mathrm{CB}$ problem. To achieve this, different possibilities for its key components were carefully designed after an in-depth analysis of the given problem. The TScB input parameter values yielding the best quality solutions to the problem at a reasonable computational effort were determined by employing a tuning methodology based on combinatorial interaction testing $[10,11]$. The performance of the new solution approach is investigated and compared with respect to a Simulated Annealing (SAcB) algorithm through extensive experimentation over a full test-suite composed of 113 benchmark graphs with a number of vertices ranging from 9 to 8192 . These graphs are divided into two different sets. The first one is composed of 85 standard graphs with known optimal solutions from seven different families including paths, cycles, meshes, trees, hypercubes and caterpillars. Certain of them were previously used to evaluate a branch \& bound (B\&B) algorithm for the CB problem [12]. The second set contains 28 graphs produced from real-world scientific and engineering applications, some of which were recently used as benchmark instances for other graph labeling problem [13, 14]. The computational results for the first set show that $\mathrm{TS}$ св attained the optimal solution for 49 graphs $(57.647 \%)$ and found good quality near-optimal solutions for the rest of the instances. For the second set, TSсв was able to reach the optimal solution for 7 instances and to ameliorate the existing theoretical upper bounds [15] for the rest of the instances. The statistical analyses performed on both experiments confirm that there exists a statistically significant increase in performance achieved by $\mathrm{TS}_{\mathrm{cB}}$ with respect to SAcB in 90 out of 113 graphs $(79.646 \%)$, highlighting the suitability of the proposed TSCB algorithm. Furthermore, these competitive results were obtained expending reasonable computational times.

The remainder of this manuscript is organized as follows. In Section 2 a brief review of some representative related work is given. The main components of the proposed TSсB algorithm are detailed in Section 3. Then, computational experiments are presented in Section 4 which are devoted to determine the best parameter settings for TSCB and to compare its performance with respect to a Simulated Annealing (SAcB) algorithm in terms of the best-known lower bounds of the state-of-the-art. Section 5 experimentally investigates the influence of some key features in the global performance of the proposed TSсв algorithm. Finally, the main conclusions of this work and some possible directions for future research are provided in Section 6. 


\section{Relevant Related Work}

The CB problem has a strong connexion with other graph embedding problems like the bandwidth minimization $[7,16]$, antibandwidth [1, 17], and cyclic antibandwidth [1].

The bandwidth minimization problem has already been described in Section 1. On the other hand, the antibandwidth and the cyclic antibandwidth problems consist in labeling the vertices of a guest graph $G$ of order $n$ by distinct vertices of either a host path $P$ or a host cycle $C$, respectively, in such a way that the minimum distance between adjacent vertices in $G$, measured in the host, is maximized.

The bandwidth problem has been the object of extensive research in the past. Different exact and heuristic algorithms for solving it have been reported in the literature. Some relevant heuristic algorithms for solving the bandwidth problem are: Tabu Search [18], GRASP with Path Relinking [19], Particle Swarm Optimization [20], Simulated Annealing [21] and Variable Neighborhood Search [22].

The work published about the antibandwidth problem was mainly devoted to find polynomial time exact algorithms for solving some special instances of the antibandwidth problem on specific classes of graphs: paths, cycles, special trees, complete and complete bipartite graphs, meshes, and tori [17, 23-27]. Some exceptions are metaheuristic algorithms including: Memetic Algorithms [28], GRASP with Path Relink [13] and Variable Neighborhood Search [14].

The cyclic antibandwidth problem has been exactly solved for some specific families of graphs like paths, cycles, two dimensional meshes and tori [29]. Asymptotic results were also obtained for hypercube and Hamming graphs $[27,30]$. However, only two metaheuristic algorithms have been reported for this important problem, a Memetic Algorithm [31] and a hybrid algorithm combining the Artificial Bee Colony methodology with Tabu Search [32].

In spite of its practical and theoretical importance, less attention has been paid to the CB problem with regard to other graph embedding problems. Up to now, most of the research on this important problem has been concentrated upon the theoretical study of its properties, with the aim of finding exact solutions for certain specific families of graphs. Next, a brief review of these studies is presented.

In 2002, Zhou proposed a systematic method for obtaining lower bounds for the bandwidth and cyclic bandwidth problems in terms of some distance- and degree-related parameters of the graph [33]. The main idea of this method is to relax the condition of embedding the graph $G$ on the host graph with the aid of a graphical parameter possessing some kind of monotonic property. This method has been demonstrated to be efficient when the parameters are chosen appropriately. The author concludes that this method yields to a number of lower bounds for the ordinary and cyclic bandwidths. In both cases, it gives rise to new estimations, as well as improvements of some known results.

Later, de Klerk et al. [34] proposed two new semidefinite programming (SDP) relaxations of the bandwidth and cyclic bandwidth based on the quadratic assignment problem (QAP) reformulation. The bounds produced by this method were tested for some special graphs showing that they are tight for paths, cliques and complete bipartite graphs. However, these bounds are not tight for hypercubes, rectangular grids and complete $k$-level $t$-ary trees.

In 1995 Yuan and Zhou [35] demonstrated that for unit interval graphs, there exists a labeling which is simultaneously optimal for the following seven labeling problems: bandwidth, cyclic bandwidth, profile, cutwidth, modified cutwidth and bandwidth sum. Following this idea, in [8] Lam et al. made a characterization of graphs with equal bandwidth and cyclic bandwidth which includes planar graphs, triangulation meshes and grids with some specific characteristics.

The CB problem has been exactly solved for twenty small instances $(n<40)$ using a branch \& bound algorithm (B\&B) recently proposed by Romero-Monsivais et al. [12]. However, this algorithm becomes impractical when the number of vertices $n$ in the studied graph increases, since the size of the search space suffers a combinatorial explosion. Therefore, there is a need for heuristic methods to address the CB problem in reasonable time.

\section{A new tabu search algorithm}

The Tabu Search (TS) algorithm was first proposed by Glover [36] and it has been widely used for solving a large number of combinatorial optimization problems [37-43]. A particularity of TS is that it explicitly employs the history of the search, both to escape from local minima and to implement an explorative strategy.

The pseudo-code of our TS implementation, called TScв, is presented in Algorithm 1. It starts with a randomly generated solution $\varphi$ (see Subsection 3.2), then it proceeds iteratively to visit a series of locally best configurations 


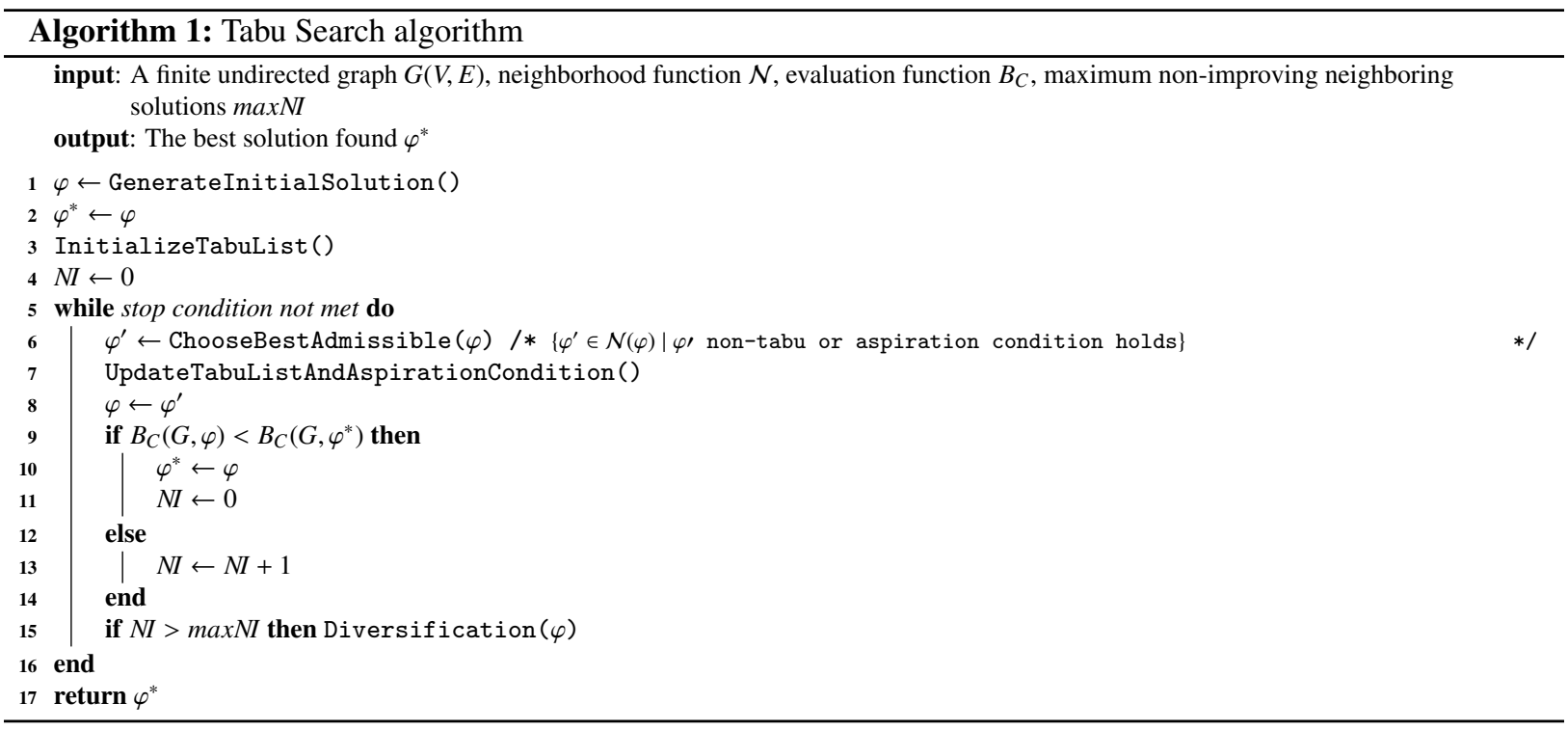

following a neighborhood function $\mathcal{N}(\varphi)$. At each iteration, a best neighbor $\varphi^{\prime}$ is chosen to replace the current configuration $\varphi$, even if the former does not improve the current one (refer to Subsection 3.3). This operation is called a move. In order to explore consecutive local optimal solutions and to avoid the occurrence of cycles, during the search, it is necessary to prohibit visiting twice the same configuration. Storing all the already visited configurations is very expensive and generally impossible in practice. An alternative approach is to only store the last moves in a recency-based memory structure, called tabu list $\mathcal{L}$ (see Subsection 3.4). The basic idea behind this memory is to record the attributes of each visited solution and to forbid the algorithm to visit again this configuration during the next $\mathcal{T}$ iterations $(\mathcal{T}$ is called the tabu tenure). In the case that more than one move have the same best cost value, one among them is randomly selected. In some cases, the tabu list may be too restrictive since certain forbidden moves could produce a solution better than the best solution found so far. To cope with this issue, an aspiration criterion is applied to accept those exceptional quality solutions (refer to Subsection 3.5).

Next, the main components of our TScв implementation are discussed in detail. For some of these components different possibilities were analyzed (see Subsection 4.4) in order to find the combination of them which offers the best quality solutions at a reasonable computational effort.

\subsection{Search space, representation and evaluation function}

Given a guest graph $G=(V, E)$ of order $|V|=n$ and $C_{n}$ a cycle graph (host) with vertex set $\left|V^{\prime}\right|=n$ and edge set $E^{\prime}$, the search space $\mathscr{E}$ for the CB problem is composed of all possible embeddings (solutions) of $G$ in $C_{n}, \varphi: V \rightarrow V^{\prime}$. Therefore, there exist $(n-1) ! / 2$ possible embeddings for a graph with $n$ vertices ${ }^{3}$.

In our TScB algorithm an embedding (labeling) $\varphi$ is represented as an array $l$ of integers with length $n$, which is indexed by the vertices and whose $i$-th value $l[i]$ denotes the label assigned to the vertex $i$. The quality $B_{C}(G, \varphi)$ of the embedding $\varphi$ is evaluated by using (1).

\subsection{Initial solution}

The initial solution is the starting embedding used for the algorithm to begin the search of better configurations in the search space $\mathscr{E}$. In this implementation the starting solution is generated randomly.

\footnotetext{
${ }^{3}$ Because each one of the $(n-1)$ ! embeddings can be reversed to obtain the same cyclic bandwidth.
} 


\subsection{Neighborhood functions}

Given that TSCB is a Local Search (LS) algorithm, then a neighborhood function must be defined. The main objective of the neighborhood function is to identify the set of potential solutions which can be reached from the current solution in an LS algorithm. Formally, a neighborhood relation is a function $\mathcal{N}: \mathscr{E} \rightarrow 2^{\mathscr{E}}$ that assigns to every potential solution (an embedding) $\varphi \in \mathscr{E}$ a set of neighboring solutions $\mathcal{N}(\varphi) \subseteq \mathscr{E}$, which is called the neighborhood of $\varphi$.

The results of our preliminary experimentations lead us to identify three suitable neighborhood structures for the $\mathrm{CB}$ problem. The logic behind these neighborhood relations, used in our TScB algorithm, is to identify those critical vertices in the graph which determine its cyclic bandwidth in order to "repair" them. These neighborhood functions are partially inspired by the work of Martí et al. on the bandwidth problem [18], which also employed a Tabu Search algorithm with a neighborhood function based on the reparation of critical vertices of the graph.

Before introducing these neighborhood structures, some preliminary concepts used in their definition are presented. Let us define the cyclic bandwidth $B_{C}(u, \varphi)$ for a vertex $u$ with respect to the embedding $\varphi$ as follows:

$$
B_{C}(u, \varphi)=\max _{v \in \mathcal{A}(u)}\left\{|\varphi(u)-\varphi(v)|_{n}\right\},
$$

where $\mathcal{A}(u)$ denotes the set of adjacent vertices of $u$, with cardinality $\operatorname{deg}(u)$. We define a vertex $u$ as critical if its cyclic bandwidth $B_{C}(u, \varphi)$ is close to $B_{C}(G, \varphi)$. Thus, the set $C(\varphi) \subseteq V$ of critical non-tabu vertices can be defined with the following expression for $0<\alpha<1$ :

$$
C(\varphi)=\left\{u \in V: B_{C}(u, \varphi) \geq \alpha B_{C}(G, \varphi), u \notin \mathcal{L}\right\}
$$

Let $\mathcal{S}(u) \subseteq \mathcal{A}(u)$ be a set of suitable swapping vertices for $u$. $\mathcal{S}(u)$ contains those non-tabu vertices adjacent to $u$ whose label values are closer to $\operatorname{mid}(u)$ than $\varphi(u)$ :

$$
\mathcal{S}(u)=\left\{v \in \mathcal{A}(u):|\operatorname{mid}(u)-\varphi(v)|_{n}<|\operatorname{mid}(u)-\varphi(u)|_{n}\right\},
$$

where $\operatorname{mid}(u)$ corresponds to the middle point of the shortest path in the host graph $C_{n}$ containing all the vertices adjacent to $u$ and is delimited by the rightmost $r(u)$ and the leftmost $l(u)$ vertices:

$$
\operatorname{mid}(u)=\left\lfloor\frac{l(u)+r(u)+a}{2}\right\rfloor \bmod n,
$$

with $a=n$, if $l(u)>r(u)$ and $a=0$ otherwise. In order to identify the values $r(u)$ and $l(u)$, the ordered sequence $\mathcal{B}=\left\{b_{1}, b_{2}, \ldots, b_{\operatorname{deg}(u)}\right\} \cup\left\{b_{1}+n\right\}$ which contains the labels currently assigned to the vertices in $\mathcal{A}(u)$ is constructed. Then, the expression:

$$
i^{*}=\underset{i \leq \operatorname{deg}(u)}{\arg \max }\left(\left(b_{i+1}-b_{i}\right)\right),
$$

is evaluated over the elements of $\mathcal{B}$ to obtain the values $l(u)=b_{i^{*}+1}$ and $r(u)=b_{i^{*}}$.

Let $\operatorname{swap}(\varphi, u, v)$ be a function allowing to exchange the labels of a pair of vertices $u$ and $v$ to produce a new embedding $\varphi^{\prime}$ where the new label for vertex $u$ is $\varphi^{\prime}(u)$, i.e., $\varphi^{\prime}(u)=\varphi(v)$ and $\varphi^{\prime}(v)=\varphi(u)$.

After introducing these preliminary concepts our first neighborhood function $\mathcal{N}_{1}(\varphi)$ can now be formally defined as follows:

$$
\mathcal{N}_{1}(\varphi)=\left\{\varphi^{\prime}=\operatorname{swap}(\varphi, u, v): u \in C(\varphi), v \in \mathcal{S}(u)\right\}
$$

It generates for every potential embedding $\varphi \in \mathscr{E}$ a set of neighboring solutions produced by exchanging the labels of a critical vertex $u \in C(\varphi)$ and the most suitable swapping vertex $v \in \mathcal{S}(u)$ for it. Every vertex $v \in \mathcal{S}$ is individually evaluated to identify the best one, i.e., the one that not only produces the smaller cyclic bandwidth $B_{C}\left(u, \varphi^{\prime}\right)$ after the application of the function $\operatorname{swap}(\varphi, u, v)$, but also the one that reduces the number of vertices adjacent to $u$ or $v$ whose cyclic bandwidth increases due to this label exchange operation. We consider that the cyclic bandwidth of a vertex $w \in \mathcal{A}(u)$ or $w \in \mathcal{A}(v)$ increased when:

$$
\begin{aligned}
& \left|\varphi^{\prime}(u)-\varphi(w)\right|_{n}>B_{C}(w, \varphi) \quad \text { and } \\
& \left|\varphi^{\prime}(u)-\varphi(w)\right|_{n}>\beta B_{C}(G, \varphi)
\end{aligned}
$$


for $0 \leq \beta \leq 1$ (acceptable cyclic bandwidth increase). If multiple vertices result into the same cyclic bandwidth $B_{C}\left(u, \varphi^{\prime}\right)$ and the same number of adjacent vertices whose cyclic bandwidth increases, then tie is broken at random.

The second neighborhood function $\mathcal{N}_{2}(\varphi, \gamma)$ is formally defined as follows:

$$
\mathcal{N}_{2}(\varphi, \gamma)=\left\{\varphi^{\prime}=\operatorname{swap}(\varphi, u, v): u \in \mathcal{C}(\varphi), v \in \mathcal{R}_{\gamma}(u)\right\}
$$

where the set $\mathcal{R}_{\gamma}(u) \subseteq V$ contains $\gamma$ non-tabu vertices randomly selected. It assigns to every potential embedding $\varphi \in \mathscr{E}$ a set of neighboring solutions which can be produced by exchanging the labels of a critical vertex $u \in C(\varphi)$ and a suitable swapping vertex $v \in \mathcal{R}_{\gamma}(u)$ for it. All the elements belonging to $\mathcal{R}_{\gamma}(u)$ are individually evaluated in order to identify the best of them. That is, the vertex $v$ that produces the smaller cyclic bandwidth $B_{C}(u, \varphi)$ when its label is exchanged with that currently assigned to vertex $u$ (ties are randomly broken).

The third neighborhood relation $\mathcal{N}_{3}(\varphi, \gamma, p)$ is a compound function partially inspired by the ideas reported in [21, 44]. It is a combination of both $\mathcal{N}_{1}(\varphi)$ and $\mathcal{N}_{2}(\varphi, \gamma)$ neighborhood functions. The former is applied with probability $(1-p)$, while the latter is employed at a $p$ rate. This combined neighborhood function $\mathcal{N}_{3}(\varphi, \gamma, p)$ is defined in (13), where $r$ is is a random number in the interval $[0,1]$.

$$
\mathcal{N}_{3}(\varphi, \gamma, p)= \begin{cases}\mathcal{N}_{1}(\varphi) & \text { if } r n d \geq p \\ \mathcal{N}_{2}(\varphi, \gamma) & \text { if } r n d<p\end{cases}
$$

\subsection{Tabu list management}

In our TScB algorithm the neighbor of a given solution $\varphi$ is obtained by exchanging the label of a critical vertex $u$ and a suitable swapping vertex $v$. When such a move is performed the vertex $u$ is classified tabu for the next $\mathcal{T}$ iterations (tabu tenure). Therefore, the label of vertex $u$ cannot be exchanged during this period.

The tabu tenure $\mathcal{T}$ for a move, in our TSCB algorithm, can be either a constant prefixed value, or it can be dynamically calculated during the search using the approach introduced by Galinier et al. [39] and used later in [45]. It is based on the use of a periodic step function $P S$ which takes as argument the number of iterations iter. Each period of this function is composed of 1500 iterations divided into 15 intervals. The value returned by $P S$ for a particular iteration iter is given by $\left(a_{j}\right)_{j=1,2, \ldots, 15}=(1,2,1,4,1,2,1,8,1,2,1,4,1,2,1) \tau$, where $\tau$ is a parameter that fixes the minimum tenure value and the index $j$ is computed with (14).

$$
j=\left\lfloor\frac{\text { iter } \bmod 1500}{100}\right\rfloor+1
$$

Therefore, the tabu tenure equals $\tau$ between iterations 1 and 99, $2 \tau$ between iterations 100 and 199, followed by $\tau$ again for iterations [200,299] and $4 \tau$ for iterations [300,399], etc. This variation scheme is periodically repeated by this function after every 1500 iterations.

\subsection{Aspiration criteria}

Since the attributes of a solution are recorded in the tabu list instead of the solutions themselves, it is possible that a candidate solution in the tabu list could lead to a better embedding than the best solution found so far. Therefore, in our TS algorithm, a simple aspiration criterion is applied: a tabu move leading to a configuration better than the best embedding found so far $\varphi^{*}$ is always accepted.

\subsection{Diversification strategy}

A diversification mechanism was also implemented since our TScB algorithm could be trapped in deep local optima. In our case, the search is judged as stagnated each time the best solution found so far $\varphi^{*}$ is not further improved after maxNI consecutive iterations. To help the search to escape from such deep local optima, a perturbation mechanism is applied to the current solution to bring diversification into the search.

The perturbation is composed by two complementary functions. The first one, called $\mathcal{D}_{1}(\varphi, \gamma)$, is defined in (15) and is applied $\rho$ consecutive times, where $\rho$ is a parameter that fixes the strength of this perturbation.

$$
\mathcal{D}_{1}(\varphi, \gamma)=\left\{\varphi^{\prime}=\operatorname{swap}(\varphi, u, v): u \in \mathcal{R}_{\gamma}(\varphi), v \in \mathcal{A}(u)\right\}
$$


It maps every potential embedding $\varphi \in \mathscr{E}$ to a set of solutions produced by exchanging the labels of a non-tabu randomly selected vertex $u \in \mathcal{R}_{\gamma}(\varphi)$ and the most appropriate adjacent vertex to it, $v \in \mathcal{A}(u)$. The most suitable vertex $v \in \mathcal{A}(u)$ for the $\operatorname{swap}(\varphi, u, v)$ is the one that results into the smaller cyclic bandwidth $B_{C}\left(u, \varphi^{\prime}\right)$ for $u$, and at the same time minimizes the number of vertices $w \in \mathcal{A}(u)$ or $w \in \mathcal{A}(v)$ whose cyclic bandwidth increases due to this label exchange operation, see (11). If there exist ties they are randomly broken.

The perturbation function $\mathcal{D}_{1}(\varphi, \gamma)$ is applied a predefined maximum number of non-consecutive times MaxS when the search stagnates. After that if the best solution found so far $\varphi^{*}$ is not further improved the second perturbation function $\mathcal{D}_{2}(\varphi, \gamma)$ is applied at most $M a x H$ non-consecutive times. This perturbation constructs a partially ordered set $\mathcal{F}(V, \leq)$ whose elements are the vertices of the graph in ascending order with respect to their cyclic bandwidth. More formally $\mathcal{F}(V, \leq)$ can be defined with the expression:

$$
\mathcal{F}(V, \leq)=\left\{u: \forall u, v \in V, B_{C}(u, \varphi) \leq B_{C}(v, \varphi)\right\}
$$

then the subset $\mathcal{H}_{\gamma} \subseteq \mathcal{F}(V, \leq)$, defined in (17), can be obtained.

$$
\mathcal{H}_{\gamma}=\left\{u_{i}: 1 \leq i \leq \gamma, u_{i} \in \mathcal{F}(V, \leq)\right\}
$$

The perturbation function $\mathcal{D}_{2}(\varphi, \gamma)$, which employs $(\gamma-1)$ consecutive times the function $\operatorname{swa} p(\varphi, u, v)$ to construct a new embedding $\varphi^{\prime}$, can be formally expressed as follows:

$$
\mathcal{D}_{2}(\varphi, \gamma)=\left\{\varphi^{\prime}=\operatorname{swap}(\varphi, u, v): u, v \in \mathcal{H}_{\gamma}\right\}
$$

\subsection{Stop condition}

The TScB algorithm stops either if a predefined maximum number of iterations (maxIter) is reached, or when the algorithm ceases to make progress. In our implementation a lack of progress exists if the perturbation function $\mathcal{D}_{2}(\varphi, \gamma)$ has been applied $M a x H$ non-consecutive times.

\subsection{Implementation considerations}

In the following section we will see that the proposed TSCB algorithm provides near-optimal solutions for the $\mathrm{CB}$ problem expending for that very reasonable computational times. This is possible thanks to the use of thoroughly designed data structures when implementing the algorithm.

For instance, in order to compute the cyclic bandwidth of an embedding $\varphi$, using the evaluation function $B_{C}(G, \varphi)$, every edge in the graph $G=(V, E)$ must be analyzed (see (1)). As a result $O(|E|)$ instructions must be executed by this complete evaluation scheme. Nevertheless, the proposed TScB algorithm employs an incremental evaluation of neighboring solutions. To this end, the cyclic distance of each edge in the graph is stored using an appropriate data structure. Indeed, suppose that the labels of two different vertices $(u, v)$ are exchanged in an embedding $\varphi$ to produce a neighboring solution $\varphi^{\prime}$, then we should only recompute the $|\mathcal{A}(u)|+|\mathcal{A}(v)|$ cyclic distances that change ${ }^{4}$ in order to obtain the cyclic bandwidth of $\varphi^{\prime}$. As it can be verified this is faster than the $O(|E|)$ operations originally required. As a consequence the TSсв algorithm is able to analyze thousands of neighboring solution employing only a very small fraction of the time that would be required by the complete evaluation scheme.

Another example of a clever data structure implemented by the $\mathrm{TS}_{\mathrm{cB}}$ algorithm is the one employed to manage the tabu list $\mathcal{L}$ (recency-based memory), which enables it to verify the tabu status of a move in constant time.

Important reductions in the total computational time expended by the proposed TScB algorithm are also possible because of the use of sorted sets in the implementation of neighborhood and diversification functions.

\section{Computational experiments}

In this section the three main experiments accomplished to evaluate the performance of the proposed TSCB algorithm, as well as that of some of its components are presented. The objective of the first experiment is to determine

\footnotetext{
${ }^{4}|\mathcal{A}(u)|$ and $|\mathcal{H}(v)|$ represent the number of adjacent vertices to $u$ and $v$, respectively.
} 
both a component combination, and a set of parameter values which permit $\mathrm{TScB}$ to attain the best trade-off between solution quality and computational effort.

The purpose of the second and third experiments is twofold: a) to carry out a performance evaluation of TScB over both a set of standard graphs with known optimal solutions and a set of graphs from real-world scientific and engineering applications; and b) to compare TScB with respect to a Simulated Annealing ( $\mathrm{SAcB}$ ) metaheuristic specially developed for the CB problem.

For all these experiments TSсB and $\mathrm{SAcB}$ were coded in $\mathrm{C}$ and compiled with $g c c$ using the optimization flag -O3. They were run sequentially into a CPU Xeon X5650 at $2.66 \mathrm{GHz}, 2$ GB of RAM with Linux operating system. Due to the non-deterministic nature of the studied algorithms, 31 independent runs were executed for each of the selected benchmark instances in each experiment presented in this section.

\subsection{Simulated annealing algorithm}

As it was mentioned in Section 2, to the best of our knowledge, there are not reported metaheuristic algorithms for the CB problem in the literature. Thus, for sake of comparison we have adapted the code of the Simulated Annealing algorithm for the bandwidth minimization problem reported in [21] to meet the special requirements of the CB problem. In particular, the new implementation, called SAcB, presents the following characteristics: a) it evaluates the fitness $B_{C}(G, \varphi)$ of the embedding $\varphi$ by employing $(1)$, b) the neighborhood $\mathcal{N}_{4}(\varphi)$ of a labeling $\varphi$ is such that for each $\varphi \in \mathscr{E}, \varphi^{\prime} \in \mathcal{N}_{4}(\varphi)$ if and only if $\varphi^{\prime}$ can be obtained by rotating the labels of any group of five consecutive vertices in the host graph $C_{n}$, c) a parameter configuration that (according to our preliminary experiments) gives a good trade-off between solution quality and computational effort: initial temperature $T_{i}=5.0$, final temperature $T_{f}=1.0 E-07$, cooling rate $c_{r}=0.97$, maximum number of visited neighboring labelings at each temperature $N V_{\max }=\lceil\max I t e r /$ decTemp $\rceil$, where maxIter $=2.0 E+07$ is a predefined global maximum number of visited neighboring labelings (iterations) and decTemp $=435$ is the total number of temperature decrements from $T_{i}$ to $T_{f}$ using a geometrical cooling scheme $T_{k}=c_{r} T_{k-1}$.

\subsection{Performance assessment and statistical significance analysis}

To evaluate the efficiency of the new proposed $\mathrm{TS} \mathrm{cB}$ implementation two criteria were selected: the best cyclic bandwidth found for each instance (smaller values are better) and the expended CPU time in seconds.

A statistical significance analysis was performed for the second and third experiments presented below. Each analysis was conducted using the following methodology. First, D'Agostino-Pearson's omnibus $K^{2}$ test was used to evaluate the normality of data distributions. For normally distributed data, either ANOVA or the Welch's $t$ parametric tests were used depending on whether the variances across the samples were homogeneous (homoskedasticity) or not. This was investigated using the Bartlett's test. For non-normal data, the nonparametric Kruskal-Wallis test was adopted. A significance level of 0.05 has been considered.

\subsection{Benchmark instances}

The performance evaluation of the new proposed TSсв implementation was carried out with extensive experiments over 113 benchmark instances ${ }^{5}$ grouped into two different sets, whose detailed descriptions are presented in the following subsections.

\subsubsection{Standard graphs}

For the first set we have generated a total of 85 standard graphs with known optimal solutions that belong to seven different families (3r-dimensional hypercubes, 10 three dimensional meshes, 12 complete $r$ level $k$-ary trees, and 15 graphs from each of the following classes: paths, cycles, two dimensional meshes and caterpillars). This set of benchmark instances is composed of 23 small graphs $(n<100), 24$ medium graphs $(100<n \leq 200)$ and 38 large graphs $(200<n \leq 8192)$. A brief description of each selected class of graph and its corresponding optimal cyclic bandwidth value are summarized below.

\footnotetext{
${ }^{5}$ Available at http://www. .cinvestav.mx/ ertello/cbmp.php
} 
1. Paths. A path graph $P_{n}$ is constructed as a linear sequence of $n$ vertices. Two of them are terminal vertices of degree one, while the others (if any) have degree two. For a path $P_{n}$ of order $n$ the optimal cyclic bandwidth value is:

$$
B_{C}\left(P_{n}\right)=1,
$$

as it was shown in [2].

2. Cycles. A cycle graph $C_{n}$ is build as a circular arrangement of $n$ vertices such that all of them have degree two. Yixun Lin [2] demonstrated that the CB problem has an optimal solution value for a cycle $C_{n}$ given by:

$$
B_{C}\left(C_{n}\right)=1 \text {. }
$$

3. Two dimensional meshes. These graphs are constructed as the Cartesian product of two paths $P_{n_{1}}$ and $P_{n_{2}}$. Hromkovič et al. [6], based on the work of Chvátalová [46], demonstrated that the optimal cyclic bandwidth for a two dimensional mesh $P_{n_{1}} \times P_{n_{2}}$ of order $n=n_{1} \cdot n_{2}$ (for $\max \left\{n_{1}, n_{2}\right\}>3$ ) is:

$$
B_{C}\left(P_{n_{1}} \times P_{n_{2}}\right)=\min \left\{n_{1}, n_{2}\right\} .
$$

4. Three dimensional meshes. A three dimensional mesh is defined as the Cartesian product of three paths. Hromkovič et al. [6] proved that the optimal cyclic bandwidth for a three dimensional mesh $P_{n} \times P_{n} \times P_{n}$ with $n^{3}$ vertices (for $n>3$ ) can be calculated with the following expression:

$$
B_{C}\left(P_{n} \times P_{n} \times P_{n}\right)=\left\lfloor\frac{3 n^{2}}{4}+\frac{n}{2}\right\rfloor .
$$

5. Complete $r$ level $k$-ary trees. These graphs are rooted complete trees in which the $i$-th level consists of $k^{i-1}$ vertices and each vertex that belongs to level $i$ has exactly $k$ descendants at level $i+1$ (for $1 \leq i<r$ ). Such a tree, denoted $T_{k, r}$, has $n=\left(k^{r}-1\right) /(k-1)$ vertices and its optimal cyclic bandwidth value is:

$$
B_{C}\left(T_{k, r}\right)=\left\lceil\frac{k\left(k^{r-1}-1\right)}{2(r-1)(k-1)}\right\rceil,
$$

see $[5,6,47]$.

6. Caterpillars. A caterpillar is a special tree in which every vertex is on a central stalk, called spine, or within distance one of the stalk, i.e., removal of its endpoints leaves a path graph. For a caterpillar $T$ with spine $P\left(u_{1}, u_{2}, \ldots, u_{m}\right)$, Lin [2] proved that the optimal cyclic bandwidth is:

$$
B_{C}(T)=\max _{1 \leq i \leq j \leq m}\left\lceil\frac{n_{i j}-1}{j-i+2}\right\rceil .
$$

where $n_{i j}$ denotes the order of the subtree $T_{i j}$ induced by $u_{i}, u_{i+1}, \ldots, u_{j}$ and all the vertices adjacent to them $(i \leq j)$.

7. $r$-dimensional hypercubes. An $r$-dimensional hypercube $Q_{r}$ is a graph usually defined as the Cartesian product of $r$ path graphs with two vertices $\left(P_{2}\right)$. It can be constructed using $n=2^{r}$ vertices labeled with $r$-bit binary numbers and connecting two vertices by an edge whenever the Hamming distance of their labels is one. Hromkovič et al. [6] proved that the optimal cyclic bandwidth for $Q_{r}(r \geq 11)$ is:

$$
B_{C}\left(Q_{r}\right)=\sum_{k=0}^{r-1}\left(\begin{array}{c}
k \\
\left\lfloor\frac{k}{2}\right\rfloor
\end{array}\right) .
$$

Although there exist other standard classes of graphs whose optimal solutions for the CB problem are reported in the literature (stars, complete and complete bipartite graphs), we have decided to not include them in this set since according to our preliminary experiments they can be solved optimally using any random labeling. In this sense these kind of instances can not be considered as good candidates for evaluating the performance of the proposed algorithm. 
Table 1: Input parameters of the TScB algorithm and their selected values.

\begin{tabular}{rrrrrrrr}
\hline & $p$ & $\mathcal{T}$ & $\max S$ & $\max H$ & $\rho$ & $\max I$ & \multicolumn{1}{c}{$\beta$} \\
\hline 0 & 0.0 & $\tau=1$ & 90 & 80 & 1 & 10 & 0.4 \\
1 & 0.2 & $\tau=2$ & 100 & 90 & 3 & 25 & 0.5 \\
2 & 0.5 & 3 & -10 & - & 5 & -100 & - \\
3 & 0.8 & 5 & - & - & - \\
\hline
\end{tabular}

\subsubsection{Harwell-Boeing graphs}

The second set contains 28 problem instances with a number of vertices between 9 and 715, coming directly from the Harwell-Boeing Sparse Matrix Collection. This collection gathers standard test matrices arising from a wide variety of scientific and engineering practical problems which can be considered as adjacency matrices in order to construct graphs. Most of the graphs in our second set (24 of them) were previously used by Duarte et al. [13] and Lozano et al. [14] as benchmarks instances for the antibandwidth problem [1]. The rest of the instances (4 graphs) were collected by us to complement this set with small graphs that can be solved exactly using the B\&B algorithm proposed in [12].

\subsection{Components and parameters tuning}

Optimizing parameter settings is an important task in the context of algorithm design. Different procedures have been proposed in the literature to find the most suitable combination of parameter values [48, 49]. In this paper we employed a tuning methodology based on combinatorial interaction testing (CIT) [10,11], which was successfully used in $[50,51]$. We have decided to use CIT, because it allows to significantly reduce the number of tests (experiments) needed to determine the best parameter settings of an algorithm. Instead of exhaustive testing all the parameter value combinations of the algorithm, it only analyzes the interactions of $t$ (or fewer) input parameters by creating interaction test-suites that include, at least once, all the $t$-way combinations between these parameters and their values.

Covering arrays (CAs) are combinatorial objects which have been extensively used to represent those interaction test-suites. A covering array, $\mathrm{CA}(N ; t, k, v)$, of size $N$, strength $t$, degree $k$, and order $v$ is an $N \times k$ array on $v$ symbols such that every $N \times t$ sub-array includes, at least once, all the ordered subsets from $v$ symbols of size $t$ ( $t$-tuples) [52]. The minimum $N$ for which a $\mathrm{CA}(N ; t, k, v)$ exists is the covering array number and it is defined according to the following expression: $\mathrm{CAN}(t, k, v)=\min \{N: \exists \mathrm{CA}(N ; t, k, v)\}$.

CAs are used to represent an interaction test-suite as follows. In an algorithm we have $k$ input parameters. Each of these has $v$ values or levels. An interaction test-suite is an $N \times k$ array where each row is a test case. Each column represents an input parameter and a value in the column is the particular configuration. This test-suite allows to cover all the $t$-way combinations of input parameter values at least once. Thus, the costs of tuning the algorithm can be substantially reduced by minimizing the number of test cases $N$ in the covering array.

In practice, algorithm's input parameters do not have exactly the same number of values (levels). To overcome this limitation of CAs, mixed level covering arrays (MCAs) are used. An $\operatorname{MCA}\left(N ; t, k,\left(v_{1}, v_{2}, \cdots, v_{k}\right)\right)$ is an $N \times k$ array on $v$ symbols $\left(v=\sum_{i=1}^{k} v_{i}\right)$, where each column $i(1 \leq i \leq k)$ of this array contains only elements from a set $S_{i}$, with $\left|S_{i}\right|=v_{i}$. This array has the property that the rows of each $N \times t$ sub-array cover all $t$-tuples of values from the $t$ columns at least once. Next, we present the details of the tuning process, based on CIT, for the particular case of our TScB algorithm.

First, we have identified $k=8$ input parameters used for TSсв: application probability for the neighborhood function $\mathcal{N}_{3}(p)$, tabu tenure $(\mathcal{T})$, maximum number of non-consecutive calls to diversification functions $\mathcal{D}_{1}(\operatorname{Max} S)$ and $\mathcal{D}_{2}($ MaxH $)$, strength of perturbation $\mathcal{D}_{1}(\rho)$, maximum number of iterations without improvement $(\max N I)$, percentage of acceptable cyclic bandwidth increase used in function $\mathcal{N}_{1}(\beta)$ and percentage $\gamma$ of vertices employed in functions $\mathcal{N}_{3}, \mathcal{D}_{1}$ and $\mathcal{D}_{2}$. Based on some preliminary experiments, certain reasonable values were selected for each one of those input parameters (shown in Table 1). No important differences in performance were observed when varying the parameter $\alpha$, which determines the number of critical vertices considered in functions $\mathcal{N}_{1}$ and $\mathcal{N}_{2}$, thus its value was fixed to 0.9 for all the experiments presented in this section.

The mixed level covering array $\operatorname{MCA}(168 ; 4,8,(4,4,3,3,3,3,2,2))$, shown (transposed) in Table 2, was obtained by using the Memetic Algorithm reported in [53]. This covering array can be easily mapped into an interaction testsuite by replacing each symbol from a column to its corresponding parameter value. For instance, we can map 0 in 
Table 2: Mixed level covering array $\operatorname{MCA}(168 ; 4,8,(4,4,3,3,3,3,2,2))$ representing an interaction test-suite for tuning TSCB (transposed).

(a) Test cases 1 to 84

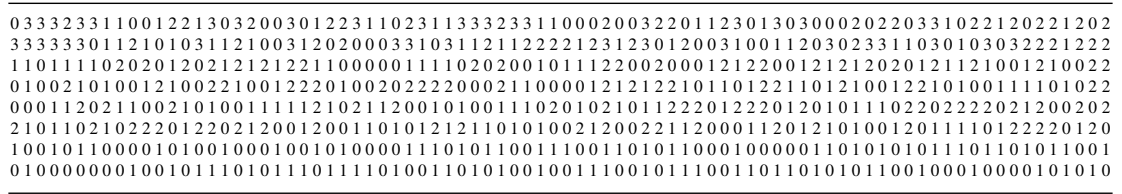

(b) Test cases 85 to 168

133312210211221321033100010303101110223320111313212003223032221330223011112310321030 31021311333210200220032023010030212232212311311302033232033301220010211033210131022 022212012120222012100211220112102211212222100102120101121010010020001022120022012222 201220002220201102202022110200210211021212121112202211101000101222100001111222200210 000001022112202211100022112211212200201100111022200022211021121220220112022000120011 010101000111111000101100100001010101001001111100111000001100010111011110111000101100 111011001101001011010010010000010101011010110001010101110101110110011001100001101000

Table 3: Results from the 10 best test cases in the tuning experiments.

\begin{tabular}{rrrrr}
\hline Num. & Test case & Avg. CB & Avg. $T$ & $R$-RMSE \\
\hline $\mathbf{7 4}$ & $\mathbf{2 0 2 0 2 1 0 1}$ & $\mathbf{4 5 . 0 0 8}$ & $\mathbf{3 6 1 . 9 2 3}$ & $\mathbf{0 . 1 6 4 6}$ \\
79 & 22212201 & 44.944 & 557.292 & 0.1648 \\
123 & 32211111 & 44.626 & 594.958 & 0.1657 \\
122 & 22122001 & 45.237 & 284.369 & 0.1682 \\
102 & 12212101 & 45.097 & 336.658 & 0.1740 \\
124 & 31222100 & 45.059 & 585.365 & 0.1743 \\
38 & 21221011 & 45.051 & 258.920 & 0.1763 \\
115 & 13112200 & 45.274 & 368.028 & 0.1767 \\
89 & 11122001 & 45.384 & 203.701 & 0.1788 \\
112 & 31112200 & 45.293 & 722.984 & 0.1792 \\
\hline
\end{tabular}

the first column (the first line in Table 2, corresponding to parameter $p$ ) to $0.0,1$ to $0.2,2$ to 0.5 and 3 to 0.8 . The resulting interaction test-suite contains, thus, 168 test cases (parameter settings) which include at least once all the 4-way combinations between TScB's input parameters and their values ${ }^{6}$.

Each one of those 168 test cases was used to run 31 times the TSCB algorithm over the 12 larger Harwell-Boeing graphs described in Subsection 4.3, resulting in a total of 62,496 executions. For each test case the relative Root Mean Square Error (R-RMSE) and the average CPU time where computed.

The R-RMSE is a quadratic scoring rule which is frequently used to measure the differences between values predicted by a model or an estimator and the values actually observed [54]. It can be formally defined using (19):

$$
\mathrm{R}-\mathrm{RMSE}=\sqrt{\frac{\sum_{i=1}^{R}\left(\frac{\hat{Y}_{i}-Y_{i}}{Y_{i}}\right)^{2}}{R}},
$$

where $\hat{Y}_{i}$ refers to the estimator of the parameter $Y_{i}$ and $R$ is the number of simulations. In optimization the R-RMSE can be used to evaluate the performance of an algorithm by measuring the differences between the solution values produced by it $\left(\hat{Y}_{i}\right)$ with respect to the best-known solutions $\left(Y_{i}\right)$ [55-57]. For a perfect performance, $\hat{Y}_{i}=Y_{i}$ and $\mathrm{R}-\mathrm{RMSE}=0$. So, the R-RMSE ranges values from 0 to infinity, with 0 corresponding to the ideal.

Table 3 summarizes the 10 test cases which yield the best results. For each test cases it lists the average cyclic bandwidth (Avg. CB), the average CPU time (Avg. T) in seconds and the R-RMSE value. This table allowed us to observe that the parameter setting giving the best trade-off between solution quality and computational effort corresponds to the test case number 74 (shown in bold). The best R-RMSE value with an acceptable speed is thus reached

\footnotetext{
${ }^{6}$ In contrast, with an exhaustive testing which contains $\left(4^{2}\right)\left(3^{4}\right)\left(2^{2}\right)=5184$ test cases
} 
Table 4: Overall performance comparison of the SAсB and $\mathrm{TS}$ св algorithms over 85 standard graphs from 7 different types all of them with known optimal solutions $B_{C}^{*}$.

\begin{tabular}{|c|c|c|c|c|c|c|c|c|c|c|}
\hline \multirow[b]{2}{*}{ Graphs } & \multirow[b]{2}{*}{ Num. } & \multicolumn{4}{|c|}{ SAсв } & \multicolumn{4}{|c|}{ TSсв } & \multirow[b]{2}{*}{$S S+$} \\
\hline & & Avg. Best & Avg. $T$ & $R-R M S E$ & $\%$ Best & Avg. Best & Avg. $T$ & $R-R M S E$ & $\%$ Best & \\
\hline path & 15 & 67.533 & 1.139 & 111.575 & 20.000 & 2.933 & 7.943 & 3.235 & 46.667 & 14 \\
\hline cycle & 15 & 67.600 & 1.086 & 112.084 & 26.667 & 2.667 & 7.219 & 3.066 & 66.667 & 13 \\
\hline mesh2D & 15 & 91.533 & 1.618 & 6.590 & 20.000 & 27.667 & 6.423 & 1.977 & 60.000 & 12 \\
\hline mesh3D & 10 & 351.600 & 3.644 & 3.836 & 0.000 & 199.900 & 17.711 & 1.853 & 10.000 & 10 \\
\hline tree & 12 & 94.917 & 0.657 & 1.416 & 16.667 & 55.250 & 5.026 & 0.034 & 83.333 & 5 \\
\hline caterpillar & 15 & 72.467 & 0.609 & 3.215 & 33.333 & 15.400 & 9.663 & 0.024 & 80.000 & 10 \\
\hline hypercube & 3 & 2387.333 & 600.000 & 1.054 & 0.000 & 1375.000 & 600.000 & 0.185 & 0.000 & 3 \\
\hline
\end{tabular}

with the following input parameter values: application probability for the neighborhood function $\mathcal{N}_{3}, p=0.5$; dynamic tabu tenure with $\tau=1$; maximum number of non-consecutive calls to diversification functions $\operatorname{MaxS}=110$ and $\mathrm{MaxH}=80$, for $\mathcal{D}_{1}$ and $\mathcal{D}_{2}$, respectively; strength of perturbation $\mathcal{D}_{1}, \rho=5$; maximum number of iterations without improvement, $\max N I=25$; percentage of acceptable cyclic bandwidth increase $\beta=0.4$ used in function $\mathcal{N}_{1}$ and percentage $\gamma=0.3$ of vertices employed in functions $\mathcal{N}_{3}, \mathcal{D}_{1}$ and $\mathcal{D}_{2}$. These values are therefore used in the experimentation reported next.

\subsection{Assessing the performance of $\mathrm{TScB}$ over standard graphs}

For the first assessing performance experiment the set of 85 standard graphs with known optimal solutions, described in Subsection 4.3.1, was selected. Then, both TSсв and SAcB were compiled and executed over these instances setting a cutoff time of 600 seconds for each execution.

The computational results produced in this experiment were first analyzed and classified by family of the tested graphs in order to separately observe the performance of the compared algorithms when solving each type of graph. Table 4 summarizes this classification. For the two compared algorithms the average (Avg. Best) of the best cyclic bandwidth cost attained by them over 31 independent executions and the average CPU time $(A v g . T)$ in seconds expended are depicted. For each class of graph tested, the relative Root Mean Square Error $(R-R M S E)$ with respect to the known optimal solution $B_{C}^{*}$ was computed using (19), as well as the percentage of instances (\%Best) for which the value of the best solution obtained by a given metaheuristic reaches $B_{C}^{*}$. Additionally, a statistical significance analysis was performed for this experiment by using the methodology described in Subsection 4.2. Last column (SS+) sums up how many times a statistically significant performance amelioration was achieved by TScB with respect to SAcB.

Table 4 shows that, for each of the seven types of standard graphs analyzed, our TScB algorithm clearly outperforms SAcв in terms of the average of the best solution cost reached (Avg. Best). Indeed, the statistical analysis carried out for this experiment established that there exists a statistically significant performance amelioration achieved by TScB with respect to SAсв on 67 benchmark instances (78.824\% of the graphs). For the other 18 tested graphs there was not a significant difference between the performance of both compared algorithms.

In general, a high $R-R M S E$ value, indicates that the solutions provided by the analyzed algorithm present a high deviation relative to the known optimal values $B_{C}^{*}$. This measure has the important property of considering a difference of only one unit with respect to an optimal value $B_{C}^{*}=1$ worse than the same one unit difference with respect to a higher $B_{C}^{*}$ value. In other words, it penalizes more the deviations with respect to small $B_{C}^{*}$ values. In this experiment, the two higher $R-R M S E$ values scored by TSCB are for the paths, and cycles, since for these types of graphs $B_{C}^{*}=1$.

Regarding the percentage of instances (\%Best) for which the best solution found by TScв equals $B_{C}^{*}$, we observe that for five types of the studied graphs (paths, cycles, two dimensional meshes, complete $r$ level $k$-ary trees and caterpillars) it is higher than $46.667 \%$ and goes up to $83.333 \%$. In the case of the three dimensional meshes and the $r$-dimensional hypercubes the \%Best value for TSсв is 10.000 and 0.000 , respectively, which reveals that these instances are challenging for TSсB, probably due to their large size and specific topology.

The detailed results from this experiment can be found in Table A.1, from which it is possible to observe that TSCB reached the optimal solution $\left(B_{C}^{*}\right)$ for $57.647 \%$ (49 out of 85 ) of the selected graphs consuming in average 29.484 seconds, which is slightly higher than the average CPU time employed by the SAcB algorithm (22.484 seconds). 


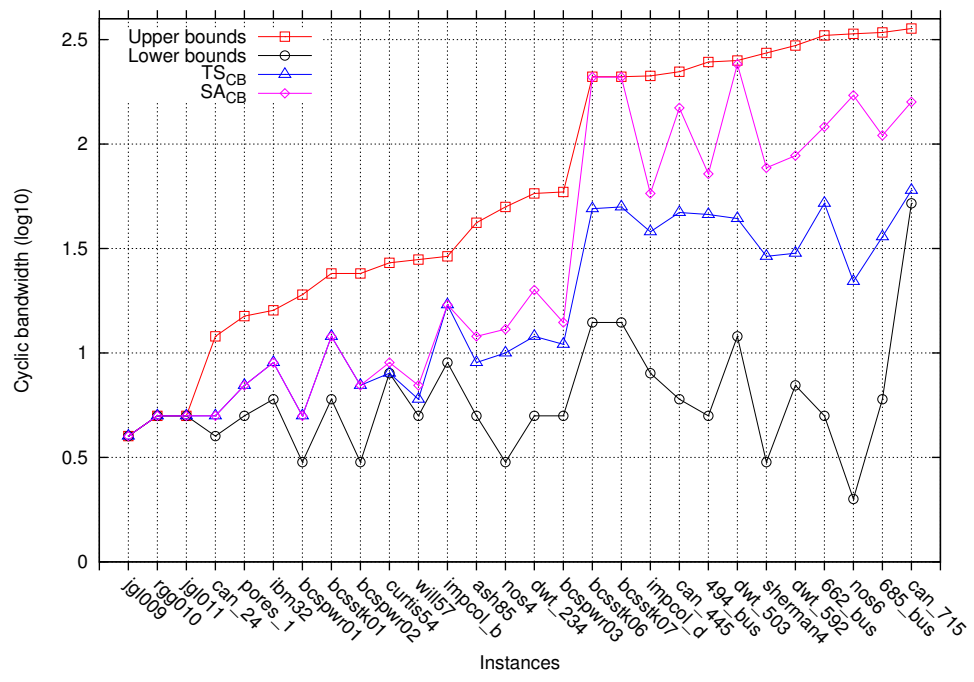

Figure 3: Performance evaluation of the best solutions achieved by SAcB and TScB, over 28 Harwell-Boeing graphs, with respect to the theoretical lower and upper bounds proposed by Lin [15].

For the other 36 benchmark instances TScB found solutions which are close to the optimal cyclic bandwidth $(R$ $R M S E=2.145)$. Another important outcome is that the solution cost found by TScB presents a relatively small standard deviation (in average 4.738). It is an indicator of the algorithm's precision and robustness since it shows that in average the performance of TScB does not present important fluctuations.

From this experiment we can conclude that TScв is certainly an effective approach for finding good quality solutions for the $\mathrm{CB}$ problem in the case of standard graphs with known tight lower bounds. Below, we will present more computational results obtained from a performance evaluation carried out with $\mathrm{TS}$ cB employing graphs produced from real-world scientific and engineering applications.

\subsection{Assessing the performance of $\mathrm{TS} \mathrm{cB}$ over Harwell-Boeing graphs}

In this experiment a performance evaluation of the best solutions achieved by $\mathrm{SAcB}$ and $\mathrm{TSCB}$ with respect to the theoretical lower and upper bounds proposed by Lin [15] was carried out over the test-suite described in Subsection 4.3.2, using a cutoff time of 600 seconds for each execution. The results from this experiment are depicted in Table A.2 using the same column headings defined at the beginning of Appendix A.

Analyzing the data presented in Table A.2 lead us to the following main observations. First, our TSCB algorithm is able to outperform the solutions provided by SAсB in 18 out of 28 graphs $(64.286 \%)$ and to equal its results for the other 10 benchmark instances. The statistical analysis presented in the last two columns of Table A.2 confirms that there exists a statistically significant increase in performance achieved by TScB with respect to SAcB on 23 graphs (82.143\% of the instances). This highlights the suitability of the studied $\mathrm{TS}$ cB approach. One can also remark that both $\mathrm{SAcB}$ and TScB are able to attain the optimal solutions, found by the B\&B algorithm reported in [12], for the 6 smallest $(n \leq 32)$ instances in this test-suite and to supply a solution cost separated only by one unit from the optimal cyclic bandwidth (5 vs. 4) for the instance bcspwr01. These results evidence that optimal solution costs could be higher than the theoretical lower bounds $\left(L_{B}\right)$ proposed by Lin [15], i.e., these lower bounds are not tight.

Second, the solution quality provided by $\mathrm{TS}$ св for the other 21 graphs (with $n \geq 39$ ) is very competitive, since it consistently improve the upper bounds $\left(U_{B}\right)$ calculated according to [15] (see Figure 3). Indeed, the $R$-RMSE for $\mathrm{TSCB}_{\mathrm{CB}}$, computed with respect to the corresponding best-known bound, is nearly one order of magnitude smaller than that of SAсв (4.055 vs. 19.174), indicating that the cost of the labelings provided by TScв are closer to the best-known solutions. Our TSсB algorithm is even able to equal the lower bound value $L_{B}=8$ for the instance curtis 54 of size $n=54$, which means that the optimal solution for this graph was found by it. Moreover, the cyclic bandwidth of the solutions reached by TScв present in average a very reduced standard deviation (see Column Dev.). 


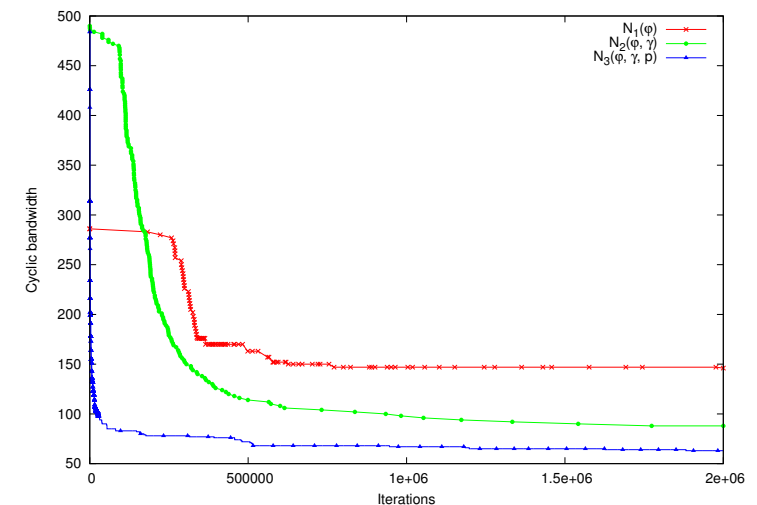

(a)

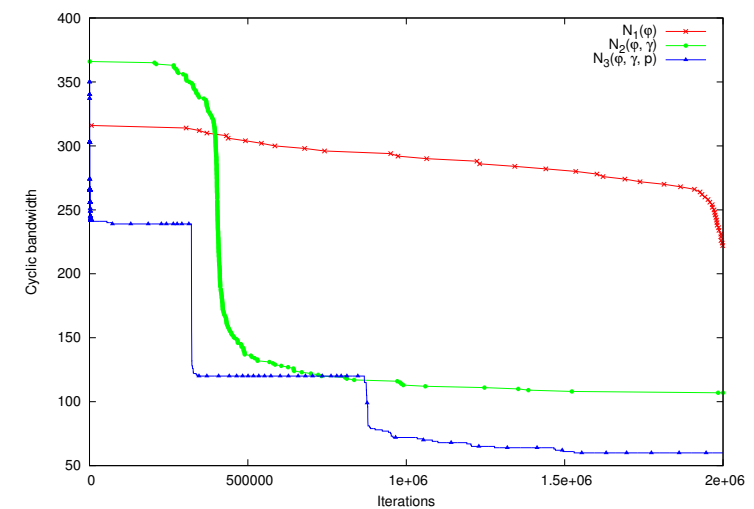

(b)

Figure 4: Average performance comparison of three neighborhood functions using TScB over the instances: (a) tree $2 x 9$ and (b) can_715.

Third, one can notice that TSсв is the most time-consuming algorithm, since it uses an average of 138.369 seconds for solving these 28 instances. On the contrary, SAсв employs only 9.515 seconds in average. However, we believe that the CPU time consumed by TScB is acceptable (at most 600 seconds) and is fully justified by considering that it is able to outperform the SAcB method in terms of cost. In addition, TSсв's consumed computing time is reasonable compared with that expended by the B\&B algorithm used in this experiment [12]. For instance, TScB employed only 600.000 seconds for finding a near-optimal solution for the larger instance in this test-suite (can_715), while the execution time for the B\&B over the instance bcspwr01 (a smaller instance) is $4.73 \mathrm{E} 05$ seconds.

\section{Discussion and analysis}

The main objective of this section is to experimentally analyze the extent to which certain key components of the proposed TSсв implementation can influence its global performance. For all the experiments presented in this section the algorithms were compiled and executed independently 31 times over the two sets of benchmark instances introduced in Subsection 4.3. The parameter values employed for TSсв were those previously identified in Subsection 4.4.

Given the space requirements for reporting the results of this experiment, our findings are presented using plots for only two representative instances. One from the set of standard graphs with known optimal solutions (tree $2 \times 9)$ and one from the set of Harwell-Boeing graphs (can_715). However, comparable results were obtained with all the other tested instances.

\subsection{Influence of the neighborhood functions}

The neighborhood function is a critical element for the performance of any local search algorithm. In order to further examine the influence of this element on the overall performance of our TScB implementation we have performed some experimental comparisons using the following three neighborhood functions (see Subsection 3.3): $\mathcal{N}_{1}(\varphi), \mathcal{N}_{2}(\varphi, \gamma)$ and $\mathcal{N}_{3}(\varphi, \gamma, p)$

For this experiment each one of the studied neighborhood functions was implemented within TScв. Figure 4 summarizes the results from this experiment. It shows three average execution (convergence) profiles which represent the evolution, during the search process (abscissa) ${ }^{7}$, of the best solution quality attained by TSCB (ordinate), when each one of the studied neighborhood relations is used to solve the instances tree $2 x 9$ and can_715. As it can be seen from the plots, the worst performance is attained by $\mathrm{TScB}$ when the neighborhood function $\mathcal{N}_{1}(\varphi)$ is used. The function $\mathcal{N}_{2}(\varphi, \gamma)$ produces better results compared with $\mathcal{N}_{1}(\varphi)$ since it improves the solution cost faster. Nevertheless, it also causes that our TSсв algorithm gets stuck on some local minima. Finally, the best performance is attained by TSCB when the function $\mathcal{N}_{3}(\varphi, \gamma, p)$ is employed, which is a compound neighborhood combining the complementary characteristics of both $\mathcal{N}_{1}(\varphi)$ and $\mathcal{N}_{2}(\varphi, \gamma)$.

${ }^{7}$ Each iteration in the plots represents a call to the evaluation function (1). 


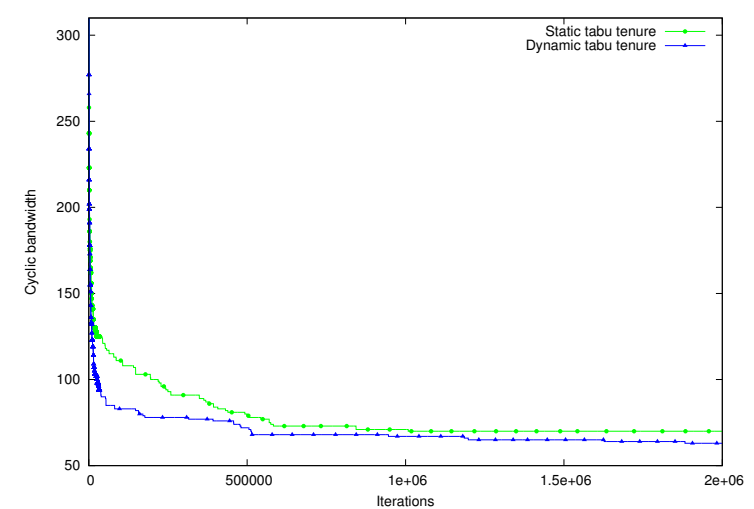

(a)

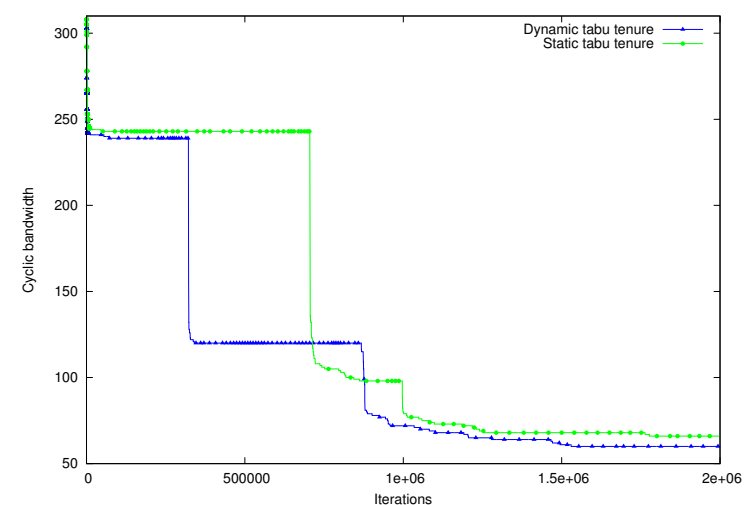

(b)

Figure 5: Average performance comparison of two different tabu tenure management strategies using TScB over the instances: (a) tree $2 x 9$ and (b) can_715.

\subsection{Influence of the tabu tenure management}

In this experiment we compare the performance of two different tabu tenure management strategies. The first one is commonly used in the literature $[36,42]$, and consists in prefixing a tabu tenure value that is maintained until the end of the algorithm's execution. The second one is the approach introduced in [39] (see Subsection 3.4), where the tabu tenure is dynamically calculated during the search using a periodic step function.

The two tabu tenure management approaches (called here Static and Dynamic, respectively) were integrated into the TSсв source code and executed for solving the selected benchmark instances. According to the results of our preliminary experiments, the tabu tenure value was fixed to $\mathcal{T}=5$ for the Static approach, while the minimum tenure value is equal to $\tau=1$ for the dynamic strategy. The results reached by TScB over the instances tree $2 x 9$ and can_715 for this comparative experiment are illustrated in Figure 5. Each plot represents the iterations of TScв (abscissa) against the average best solution quality attained with the used of the compared tabu tenure management mechanism (ordinate). This figure discloses that $\mathrm{TS}_{\mathrm{cB}}$ using a tabu tenure value which is dynamically calculated throughout the search, with a periodic step function, performs slightly better than the TSсв implementation that prefixes a tabu tenure value. Very similar results were obtained with the rest of the analyzed benchmark instances, thus this figure correctly summarizes the behavior of the compared tabu tenure management schemes.

\subsection{Influence of the diversification strategy}

The diversification strategy is an important component that must be carefully designed when implementing a tabu search algorithm, since it is related with the process of discovering new unexplored regions of the search space containing potentially good solutions $[58,59]$. This experiment is thus devoted to investigate the extent to which this key component of TScB can influence its global performance.

Two different versions of the $\mathrm{TS}_{\mathrm{cB}}$ algorithm were specially prepared to this end. The first one equipped with the diversification strategy described in Subsection 3.6. The second one without any diversification strategy implemented. Both versions were executed independently over the selected benchmark instances. Figure 6 displays, for the instances tree $2 \times 9$ and can_715, two convergence profiles representing the evolution through the search (abscissa) of the current and best solution quality provided by the $\mathrm{TS}$ с algorithm employing the proposed diversification strategy (ordinate). The iteration when the perturbation function $\mathcal{D}_{2}(\varphi, \gamma)$ is applied to diversify the search is also marked in these plots with a symbol " $x$ ". Recall, that $\mathcal{D}_{2}(\varphi, \gamma)$ is only applied after a predefined maximum number $($ Max $S)$ of non-consecutive calls to the perturbation function $\mathcal{D}_{1}(\varphi, \gamma)$. For comparative purposes, the best solution quality attained by the TSсB version without any diversification strategy implemented is additionally presented in a third plot. From this figure it can be easily observed that the TSсв algorithm who does not implement a diversification strategy has the worst overall performance in this experiment. This point can be better explained by observing that the lack of a pertinent diversification strategy causes that the TSсв algorithm easily stagnates in deep local optima. On the contrary, the TSсв algorithm employing the proposed diversification strategy is able to detect when the search process 


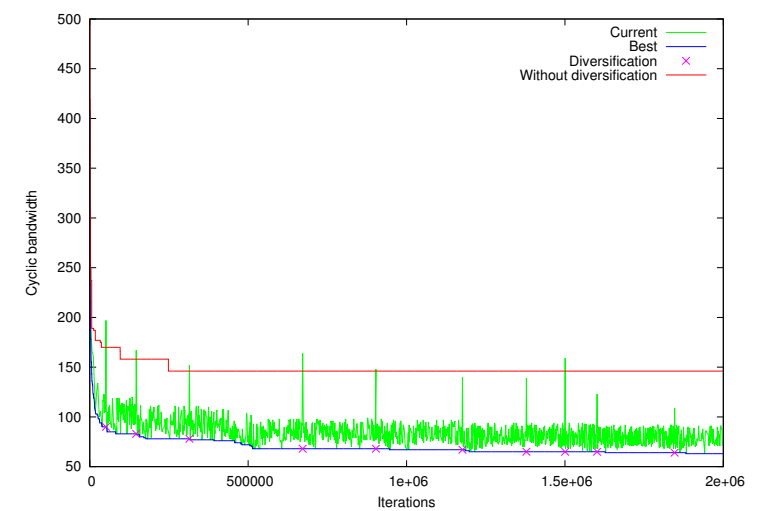

(a)

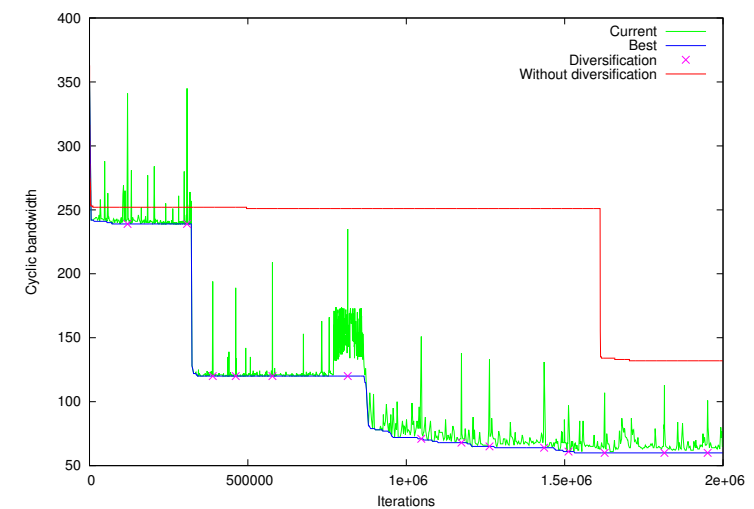

(b)

Figure 6: Performance comparison of TSCB with and without a diversification strategy over the instances: (a) tree $2 x 9$ and (b) can_715.

is trapped into some local minima and to guide it to more promising potential solutions. This is confirmed by the fact that $\mathrm{TS}_{\mathrm{cB}}$ with a diversification mechanism consistently attains better global performance in terms of cyclic bandwidth cost.

\section{Conclusions}

In this paper the first implementation of a Tabu Search algorithm ( $\left.\mathrm{TS}_{\mathrm{cB}}\right)$ for solving the Cyclic Bandwidth problem (CB) was presented. This algorithm integrates two key features that importantly determine its performance. First, a carefully designed composed neighborhood function which allows the search to quickly reduce the total cost of candidate solutions, while avoiding to get stuck on some local minima. Second, an effective tabu list management method where the tabu tenure for a move is dynamically calculated during the search using a specially designed periodic step function.

TScв's components and parameter values were carefully determined, through the use of a tuning methodology based on combinatorial interaction testing $[10,11]$, to yield the best solution quality in a reasonable computational time. Then, the practical effectiveness of TSсB was assessed through extensive experimentation over a set of 113 standard benchmark instances [12-14], and compared against a Simulated Annealing implementation (SACB).

The first of these experiments employed 85 standard graphs with known optimal solutions to carefully compare the performance of both $\mathrm{SAcB}$ and $\mathrm{TS}$ св with respect to the known optimal solutions $\left(B_{C}^{*}\right)$. This experiment demonstrated that there exists a statistically significant performance amelioration achieved by TSCB with respect to SAcB in 67 out of 85 tested graphs $(78.824 \%)$. Our proposed method attained the optimal solutions value $B_{C}^{*}$ for 49 out of $85(57.647 \%)$ selected graphs expending in average 29.484 seconds, which is slightly higher than the average CPU time employed by the SAcB algorithm (22.484 seconds). For the rest of the graphs TScB found good quality solutions, which are close to the optimal cyclic bandwidth $(R-R M S E=2.145$ in average).

The second experiment aimed at performing a comparative evaluation of the best solutions achieved by SAсв and $\mathrm{TSCB}_{\mathrm{C}}$ with respect to the theoretical bounds proposed by Lin [15] for the CB problem. For this experiment 28 test instances produced from real-world scientific and engineering applications (Harwell-Boeing graphs) were used. The obtained results confirm that TScB's performance was statistically superior than that reached by SAcB on $82.143 \%$ of the tested instances (23 out of 28). In fact, TSсB was able to find the optimal solution $B_{C}^{*}$ for 7 graphs in this test-suite and to establish new better upper bounds for the other 21 instances.

The third experiment was devoted to experimentally analyze the extent to which some key components of the proposed TSсв implementation (neighborhood function, tabu tenure management and diversification strategy) can influence its convergence process. Thanks to this analysis it was possible to identify that the use of the proposed compound neighborhood function coupled with a diversification strategy are the two key components which determine the global performance of our $\mathrm{TS}_{\mathrm{cB}}$ algorithm. The compound neighborhood function permits a fast improving of the solution cost while avoiding to get stuck on some local minima. The diversification strategy, on the other hand, allows 
the TScв algorithm to detect when the search process is trapped into some local minima and to guide it to more promising potential solutions, which results in a better global performance in terms of cyclic bandwidth cost.

All the experimental results presented confirm the practical advantages of using our TScB algorithm for solving the $\mathrm{CB}$ problem. It is a robust algorithm yielding good quality solutions for the CB problem in the case of general graphs at competitive computational time. In this sense this work represents an original contribution in this field.

Although promising results were obtained by the $\mathrm{TS} \mathrm{CB}$ algorithm, we believe that they could be still ameliorated. Our future work will concentrate on designing and evaluating alternative neighborhood and evaluation functions in order to boost the performance of the proposed TScB algorithm, since it is well-known that they are two important components which define the so-called landscape of the search problem [60,61] and impact thus greatly the efficiency of the search algorithms [62].

\section{Acknowledgements}

The authors thankfully acknowledge the high performance computing resources (Neptuno cluster) and the technical assistance provided by the Information Technology Laboratory at CINVESTAV-Tamaulipas. The four reviewers of the paper are greatly acknowledged for their constructive comments which have aided to improve the presentation of this paper.

\section{A. Detailed comparison of the SACB and TScB algorithms}

Tables A.1 and A.2 present detailed results from the experimental comparison carried out between the TSCB and SAcB algorithms over both a set of standard graphs with known optimal solutions and a set of graphs from real-world scientific and engineering applications. The first three columns in these tables represent the graph name, its number of vertices $(|V|)$ and edges $(|E|)$. In Table A.1 the known optimal solution $\left(B_{C}^{*}\right)$ was calculated using equations presented in Subsection 4.3.1. The theoretical lower $\left(L_{B}\right)$ and upper $\left(U_{B}\right)$ bounds for the graphs listed in Table A.2 (see Columns 4 and 5) were computed, as indicated by Lin [15], with the expressions $L_{B}=\lceil\Delta(G) / 2\rceil$ and $U_{B}=\lfloor|V| / 2\rfloor$, where $\Delta(G)$ denotes the maximum degree of the graph $G$. For the first seven instances (with $n \leq 40$ ) in Table A.2, the exact optimal solutions $B_{C}^{*}$ were obtained using the $\mathrm{B} \& \mathrm{~B}$ algorithm reported in [12]. In both tables, for each compared algorithm five columns are used to depict the best (Best), average (Avg.) and standard deviation (Dev.) of the cyclic bandwidth cost reached by that method over 31 independent executions, its average CPU time $(T)$ in seconds, and the difference $(D)$ between its best result (Best) and the corresponding best-known bound (either $B_{C}^{*}$ or $L_{B}$ ). A statistical significance analysis was performed for these experiments by using the methodology described in Subsection 4.2 and the resulting $p$-value is presented. If a statistically significant difference exists between the performance of TSCB and SAcB, the corresponding cells in the last column $(S S)$ are marked either "+" or "-" depending on whether such a difference favors TSCB or not. Unmarked cells indicate that there was not a significant difference between the compared algorithms.

Table A.1: Detailed performance assessment of the SAcB and TScB algorithms over 85 standard graphs from 7 different types all of them with known optimal solutions $B_{C}^{*}$.

\begin{tabular}{|c|c|c|c|c|c|c|c|c|c|c|c|c|c|c|c|}
\hline \multirow[b]{2}{*}{ Graph } & \multirow[b]{2}{*}{$|V|$} & \multirow[b]{2}{*}{$|E|$} & \multirow[b]{2}{*}{$B_{C}^{*}$} & \multicolumn{5}{|c|}{ SAcB } & \multicolumn{5}{|c|}{$\mathrm{TS} \mathrm{CB}_{\mathrm{B}}$} & \multirow[b]{2}{*}{ p-value } & \multirow[b]{2}{*}{$S S$} \\
\hline & & & & Best & Avg. & Dev. & $T$ & $D$ & Best & Avg. & Dev. & $T$ & $D$ & & \\
\hline path20 & 20 & 19 & 1 & 1 & 1.000 & 0.000 & 1.665 & 0 & 1 & 1.000 & 0.000 & 0.133 & 0 & $1.0 \mathrm{E}+00$ & \\
\hline path25 & 25 & 24 & 1 & 1 & 1.500 & 0.527 & 1.876 & 0 & 1 & 1.000 & 0.000 & 0.603 & 0 & 1.2E-02 & + \\
\hline path30 & 30 & 29 & 1 & 1 & 1.600 & 0.843 & 3.107 & 0 & 1 & 1.000 & 0.000 & 1.422 & 0 & $3.0 \mathrm{E}-02$ & + \\
\hline path35 & 35 & 34 & 1 & 2 & 2.600 & 0.516 & 3.292 & 1 & 1 & 1.000 & 0.000 & 3.420 & 0 & $3.8 \mathrm{E}-05$ & + \\
\hline path40 & 40 & 39 & 1 & 2 & 2.800 & 0.422 & 3.711 & 1 & 1 & 1.000 & 0.000 & 3.330 & 0 & $2.7 \mathrm{E}-05$ & + \\
\hline path100 & 100 & 99 & 1 & 15 & 18.500 & 2.173 & 0.030 & 14 & 1 & 1.500 & 0.527 & 1.920 & 0 & $1.4 \mathrm{E}-16$ & + \\
\hline path125 & 125 & 124 & 1 & 21 & 25.100 & 3.348 & 0.037 & 20 & 1 & 1.700 & 0.483 & 5.865 & 0 & 4.6E-18 & + \\
\hline path150 & 150 & 149 & 1 & 28 & 31.000 & 2.108 & 0.055 & 27 & 2 & 2.000 & 0.000 & 3.295 & 1 & $6.6 \mathrm{E}-13$ & + \\
\hline path175 & 175 & 174 & 1 & 31 & 35.300 & 2.111 & 0.083 & 30 & 2 & 2.000 & 0.000 & 6.836 & 1 & $5.0 \mathrm{E}-05$ & + \\
\hline path200 & 200 & 199 & 1 & 40 & 42.300 & 2.791 & 0.103 & 39 & 2 & 2.000 & 0.000 & 9.612 & 1 & 4.1E-05 & + \\
\hline path300 & 300 & 299 & 1 & 63 & 67.400 & 3.134 & 0.191 & 62 & 3 & 3.200 & 0.422 & 10.470 & 2 & $6.8 \mathrm{E}-05$ & + \\
\hline path475 & 475 & 474 & 1 & 115 & 120.600 & 4.300 & 0.375 & 114 & 5 & 5.600 & 0.516 & 14.566 & 4 & $1.2 \mathrm{E}-04$ & + \\
\hline
\end{tabular}


Table A.1 - Continued from previous page

\begin{tabular}{|c|c|c|c|c|c|c|c|c|c|c|c|c|c|c|c|}
\hline \multirow[b]{2}{*}{ Graph } & \multirow[b]{2}{*}{$|V|$} & \multirow[b]{2}{*}{$|E|$} & \multirow[b]{2}{*}{$B_{C}^{*}$} & \multicolumn{5}{|c|}{ SAсв } & \multicolumn{5}{|c|}{$\mathrm{TS}_{\mathrm{CB}}$} & \multirow[b]{2}{*}{ p-value } & \multirow[b]{2}{*}{$S S$} \\
\hline & & & & Best & Avg. & Dev. & $T$ & $D$ & Best & Avg. & Dev. & $T$ & $D$ & & \\
\hline path650 & 650 & 649 & 1 & 171 & 176.700 & 4.473 & 0.592 & 170 & 7 & 7.000 & 0.000 & 17.428 & 6 & $7.0 \mathrm{E}-05$ & + \\
\hline path825 & 825 & 824 & 1 & 229 & 237.600 & 3.373 & 0.870 & 228 & 8 & 8.000 & 0.000 & 19.831 & 7 & $3.9 \mathrm{E}-14$ & + \\
\hline path1000 & 1000 & 999 & 1 & 293 & 300.600 & 5.082 & 1.104 & 292 & 8 & 8.900 & 0.316 & 20.409 & 7 & $5.0 \mathrm{E}-15$ & + \\
\hline cycle 20 & 20 & 20 & 1 & 1 & 1.300 & 0.483 & 1.746 & 0 & 1 & 1.000 & 0.000 & 0.356 & 0 & $6.7 \mathrm{E}-02$ & \\
\hline cycle25 & 25 & 25 & 1 & 1 & 1.100 & 0.316 & 2.005 & 0 & 1 & 1.000 & 0.000 & 0.285 & 0 & 3.2E-01 & \\
\hline cycle30 & 30 & 30 & 1 & 1 & 2.400 & 0.966 & 2.210 & 0 & 1 & 1.000 & 0.000 & 0.573 & 0 & $1.4 \mathrm{E}-03$ & + \\
\hline cycle35 & 35 & 35 & 1 & 1 & 2.400 & 0.843 & 3.315 & 0 & 1 & 1.000 & 0.000 & 0.571 & 0 & $5.1 \mathrm{E}-04$ & + \\
\hline cycle40 & 40 & 40 & 1 & 2 & 3.000 & 0.816 & 3.535 & 1 & 1 & 1.000 & 0.000 & 0.548 & 0 & 4.7E-05 & + \\
\hline cycle 100 & 100 & 100 & 1 & 16 & 19.200 & 3.393 & 0.031 & 15 & 1 & 1.000 & 0.000 & 1.898 & 0 & $4.0 \mathrm{E}-05$ & + \\
\hline cycle 125 & 125 & 125 & 1 & 22 & 24.500 & 1.841 & 0.047 & 21 & 1 & 1.000 & 0.000 & 1.920 & 0 & $3.8 \mathrm{E}-05$ & + \\
\hline cycle 150 & 150 & 150 & 1 & 25 & 29.000 & 3.127 & 0.067 & 24 & 1 & 1.000 & 0.000 & 3.300 & 0 & $3.8 \mathrm{E}-05$ & + \\
\hline cycle175 & 175 & 175 & 1 & 32 & 35.600 & 2.797 & 0.085 & 31 & 1 & 1.000 & 0.000 & 8.192 & 0 & $4.8 \mathrm{E}-05$ & + \\
\hline cycle 200 & 200 & 200 & 1 & 38 & 40.200 & 1.317 & 0.114 & 37 & 1 & 1.000 & 0.000 & 9.254 & 0 & $5.0 \mathrm{E}-05$ & + \\
\hline cycle 300 & 300 & 300 & 1 & 64 & 67.300 & 2.263 & 0.186 & 63 & 3 & 3.100 & 0.316 & 11.528 & 2 & 7.1E-05 & + \\
\hline cycle 475 & 475 & 475 & 1 & 113 & 119.800 & 4.780 & 0.388 & 112 & 5 & 5.800 & 0.422 & 14.703 & 4 & $3.8 \mathrm{E}-15$ & + \\
\hline cycle 650 & 650 & 650 & 1 & 170 & 178.100 & 4.067 & 0.605 & 169 & 7 & 7.600 & 0.516 & 16.348 & 6 & $3.4 \mathrm{E}-16$ & + \\
\hline cycle825 & 825 & 825 & 1 & 237 & 241.500 & 3.749 & 0.885 & 236 & 7 & 7.900 & 0.316 & 18.262 & 6 & 7.3E-05 & + \\
\hline cycle1000 & 1000 & 1000 & 1 & 291 & 298.700 & 5.438 & 1.071 & 290 & 8 & 8.500 & 0.850 & 20.542 & 7 & $1.3 \mathrm{E}-20$ & + \\
\hline mesh2D $5 \times 4$ & 20 & 31 & 4 & 4 & 4.800 & 0.422 & 1.829 & 0 & 4 & 4.000 & 0.000 & 2.291 & 0 & 3.7E-04 & + \\
\hline mesh2D5x5 & 25 & 40 & 5 & 5 & 5.000 & 0.000 & 3.352 & 0 & 5 & 5.000 & 0.000 & 2.932 & 0 & $1.0 \mathrm{E}+00$ & \\
\hline mesh2D5x6 & 30 & 49 & 5 & 5 & 6.200 & 1.317 & 4.702 & 0 & 5 & 5.000 & 0.000 & 1.633 & 0 & $5.0 \mathrm{E}-03$ & + \\
\hline $\operatorname{mesh} 2 \mathrm{D} 5 \mathrm{x} 7$ & 35 & 58 & 5 & 6 & 7.100 & 1.449 & 2.790 & 1 & 5 & 5.000 & 0.000 & 1.393 & 0 & $4.0 \mathrm{E}-05$ & + \\
\hline mesh2D5x8 & 40 & 67 & 5 & 6 & 6.500 & 0.972 & 4.401 & 1 & 5 & 5.000 & 0.000 & 1.770 & 0 & $3.5 \mathrm{E}-05$ & + \\
\hline $\operatorname{mesh} 2 \mathrm{D} 10 \times 10$ & 100 & 180 & 10 & 26 & 28.000 & 2.309 & 0.047 & 16 & 10 & 10.500 & 0.527 & 3.832 & 0 & $5.6 \mathrm{E}-05$ & + \\
\hline mesh2D $5 \times 25$ & 125 & 220 & 5 & 28 & 33.500 & 2.415 & 0.090 & 23 & 5 & 5.000 & 0.000 & 2.790 & 0 & $2.0 \mathrm{E}-05$ & + \\
\hline mesh2D10x15 & 150 & 275 & 10 & 39 & 42.400 & 3.596 & 0.121 & 29 & 11 & 11.000 & 0.000 & 3.031 & 1 & $2.0 \mathrm{E}-05$ & + \\
\hline mesh2D $7 \times 25$ & 175 & 318 & 7 & 45 & 47.200 & 1.751 & 0.179 & 38 & 7 & 8.700 & 3.335 & 6.183 & 0 & $6.3 \mathrm{E}-05$ & + \\
\hline mesh2D8x25 & 200 & 367 & 8 & 52 & 53.700 & 1.059 & 0.231 & 44 & 8 & 8.200 & 0.422 & 3.588 & 0 & 7.2E-05 & + \\
\hline mesh2D $15 \times 20$ & 300 & 565 & 15 & 81 & 88.800 & 3.553 & 0.434 & 66 & 16 & 33.900 & 28.368 & 6.330 & 1 & 4.1E-05 & + \\
\hline mesh2D19x25 & 475 & 906 & 19 & 154 & 160.200 & 4.614 & 0.824 & 135 & 119 & 119.900 & 0.316 & 9.267 & 100 & $1.8 \mathrm{E}-01$ & \\
\hline mesh2D $25 \times 26$ & 650 & 1249 & 25 & 233 & 236.600 & 4.061 & 1.246 & 208 & 164 & 164.000 & 0.000 & 14.154 & 139 & $1.9 \mathrm{E}-04$ & + \\
\hline mesh2D 28x30 & 840 & 1622 & 28 & 313 & 317.300 & 3.860 & 1.824 & 285 & 30 & 174.000 & 75.895 & 15.557 & 2 & $1.2 \mathrm{E}-01$ & \\
\hline mesh2D20x50 & 1000 & 1930 & 20 & 376 & 386.600 & 6.484 & 2.206 & 356 & 21 & 113.100 & 117.825 & 21.600 & 1 & $1.0 \mathrm{E}-03$ & + \\
\hline mesh3D4 & 64 & 300 & 14 & 19 & 20.700 & 0.949 & 0.032 & 5 & 16 & 16.000 & 0.000 & 0.060 & 2 & $1.3 \mathrm{E}-05$ & + \\
\hline mesh3D5 & 125 & 540 & 21 & 34 & 38.500 & 2.718 & 0.168 & 13 & 21 & 21.700 & 0.483 & 0.260 & 0 & 4.7E-04 & + \\
\hline mesh3D6 & 216 & 882 & 30 & 62 & 76.700 & 6.897 & 0.370 & 32 & 31 & 31.000 & 0.000 & 6.578 & 1 & 7.7E-05 & + \\
\hline mesh3D7 & 343 & 1344 & 40 & 118 & 127.700 & 5.143 & 0.785 & 78 & 42 & 42.000 & 0.000 & 5.510 & 2 & $8.5 \mathrm{E}-05$ & + \\
\hline mesh3D8 & 512 & 1344 & 52 & 200 & 207.400 & 3.239 & 1.341 & 148 & 129 & 129.600 & 0.516 & 17.460 & 77 & $2.1 \mathrm{E}-02$ & + \\
\hline mesh3D9 & 729 & 1944 & 65 & 306 & 308.800 & 1.619 & 2.065 & 241 & 184 & 184.700 & 0.483 & 19.195 & 119 & $7.8 \mathrm{E}-14$ & + \\
\hline mesh3D10 & 1000 & 2700 & 80 & 427 & 432.300 & 4.523 & 3.399 & 347 & 252 & 253.100 & 0.568 & 23.299 & 172 & 8.2E-09 & + \\
\hline mesh3D11 & 1331 & 3630 & 96 & 582 & 588.500 & 2.799 & 5.908 & 486 & 336 & 336.800 & 0.422 & 28.427 & 240 & $4.2 \mathrm{E}-11$ & + \\
\hline mesh3D12 & 1728 & 4752 & 114 & 772 & 778.900 & 5.626 & 8.994 & 658 & 435 & 435.800 & 0.422 & 35.164 & 321 & $1.7 \mathrm{E}-16$ & + \\
\hline mesh3D13 & 2197 & 6084 & 133 & 996 & 1002.600 & 3.658 & 13.382 & 863 & 553 & 553.600 & 0.699 & 41.161 & 420 & $6.3 \mathrm{E}-23$ & + \\
\hline tree $2 \times 4$ & 31 & 30 & 4 & 4 & 4.000 & 0.000 & 2.617 & 0 & 4 & 4.000 & 0.000 & 0.502 & 0 & $1.0 \mathrm{E}+00$ & \\
\hline tree $3 \times 3$ & 40 & 39 & 7 & 7 & 7.000 & 0.000 & 1.927 & 0 & 7 & 7.000 & 0.000 & 0.314 & 0 & $1.0 \mathrm{E}+00$ & \\
\hline tree $10 \mathrm{x} 2$ & 111 & 110 & 28 & 29 & 30.300 & 1.160 & 0.029 & 1 & 28 & 28.000 & 0.000 & 0.003 & 0 & $1.0 \mathrm{E}+00$ & \\
\hline tree $3 \times 4$ & 121 & 120 & 15 & 23 & 24.200 & 0.919 & 0.039 & 8 & 15 & 15.700 & 0.483 & 0.538 & 0 & 7.3E-07 & + \\
\hline tree $5 \times 3$ & 156 & 155 & 26 & 33 & 36.900 & 1.912 & 0.049 & 7 & 26 & 26.000 & 0.000 & 5.869 & 0 & $3.7 \mathrm{E}-04$ & + \\
\hline tree $13 \times 2$ & 183 & 182 & 46 & 47 & 48.400 & 1.955 & 0.071 & 1 & 46 & 46.000 & 0.000 & 0.067 & 0 & $1.0 \mathrm{E}+00$ & \\
\hline tree $2 \times 7$ & 255 & 254 & 19 & 52 & 60.900 & 3.985 & 0.148 & 33 & 20 & 20.100 & 0.316 & 7.306 & 1 & 3.6E-09 & + \\
\hline tree $17 x 2$ & 307 & 306 & 77 & 83 & 88.900 & 3.213 & 0.196 & 6 & 77 & 77.000 & 0.000 & 0.525 & 0 & $1.0 \mathrm{E}+00$ & \\
\hline tree $21 \times 2$ & 463 & 462 & 116 & 133 & 139.700 & 3.529 & 0.376 & 17 & 116 & 116.000 & 0.000 & 0.811 & 0 & $1.0 \mathrm{E}+00$ & \\
\hline tree $25 \times 2$ & 651 & 650 & 163 & 203 & 207.400 & 3.658 & 0.620 & 40 & 163 & 163.000 & 0.000 & 1.098 & 0 & $3.2 \mathrm{E}-01$ & \\
\hline tree $5 \times 4$ & 781 & 780 & 98 & 219 & 229.800 & 5.884 & 0.716 & 121 & 98 & 98.200 & 0.422 & 19.670 & 0 & $6.0 \mathrm{E}-12$ & + \\
\hline tree $2 \times 9$ & 1023 & 1022 & 57 & 306 & 316.000 & 6.254 & 1.099 & 249 & 63 & 64.200 & 0.919 & 23.608 & 6 & $2.4 \mathrm{E}-15$ & + \\
\hline caterpillar3 & 9 & 8 & 3 & 3 & 3.000 & 0.000 & 0.000 & 0 & 3 & 3.000 & 0.000 & 20.046 & 0 & $1.0 \mathrm{E}+00$ & \\
\hline caterpillar4 & 14 & 13 & 3 & 3 & 3.000 & 0.000 & 0.368 & 0 & 3 & 3.000 & 0.000 & 0.329 & 0 & $1.0 \mathrm{E}+00$ & \\
\hline caterpillar5 & 20 & 19 & 4 & 4 & 4.000 & 0.000 & 1.480 & 0 & 4 & 4.000 & 0.000 & 0.719 & 0 & $1.0 \mathrm{E}+00$ & \\
\hline caterpillar6 & 27 & 26 & 5 & 5 & 5.000 & 0.000 & 1.644 & 0 & 5 & 5.000 & 0.000 & 0.540 & 0 & $1.0 \mathrm{E}+00$ & \\
\hline caterpillar7 & 35 & 34 & 6 & 6 & 6.000 & 0.000 & 1.809 & 0 & 6 & 6.000 & 0.000 & 0.765 & 0 & $1.0 \mathrm{E}+00$ & \\
\hline caterpillar13 & 104 & 103 & 10 & 16 & 20.400 & 3.307 & 0.032 & 6 & 10 & 10.000 & 0.000 & 4.058 & 0 & $2.0 \mathrm{E}-05$ & + \\
\hline caterpillar14 & 119 & 118 & 11 & 20 & 25.000 & 4.190 & 0.036 & 9 & 11 & 11.000 & 0.000 & 5.391 & 0 & $3.4 \mathrm{E}-05$ & + \\
\hline caterpillar16 & 152 & 151 & 13 & 30 & 33.800 & 4.467 & 0.065 & 17 & 13 & 13.000 & 0.000 & 6.835 & 0 & $3.4 \mathrm{E}-05$ & + \\
\hline caterpillar17 & 170 & 169 & 14 & 32 & 36.000 & 2.749 & 0.085 & 18 & 14 & 14.000 & 0.000 & 4.902 & 0 & $3.8 \mathrm{E}-05$ & + \\
\hline caterpillar19 & 209 & 208 & 15 & 41 & 45.200 & 1.751 & 0.121 & 26 & 15 & 15.900 & 0.316 & 9.500 & 0 & $1.1 \mathrm{E}-04$ & + \\
\hline
\end{tabular}


Table A.1 - Continued from previous page

\begin{tabular}{|c|c|c|c|c|c|c|c|c|c|c|c|c|c|c|c|}
\hline \multirow[b]{2}{*}{ Graph } & \multirow[b]{2}{*}{$|V|$} & \multirow[b]{2}{*}{$|E|$} & \multirow[b]{2}{*}{$B_{C}^{*}$} & \multicolumn{5}{|c|}{ SAcв } & \multicolumn{5}{|c|}{$\mathrm{TS} \mathrm{cB}$} & \multirow[b]{2}{*}{$p$-value } & \multirow[b]{2}{*}{$S S$} \\
\hline & & & & Best & Avg. & Dev. & $T$ & $D$ & Best & Avg. & Dev. & $T$ & $D$ & & \\
\hline caterpillar23 & 299 & 298 & 19 & 65 & 72.500 & 4.478 & 0.181 & 46 & 19 & 19.300 & 0.483 & 13.259 & 0 & $2.0 \mathrm{E}-09$ & + \\
\hline caterpillar29 & 464 & 463 & 24 & 118 & 124.100 & 3.604 & 0.358 & 94 & 24 & 25.800 & 0.919 & 15.077 & 0 & $3.9 \mathrm{E}-14$ & + \\
\hline caterpillar35 & 665 & 664 & 29 & 182 & 196.000 & 8.524 & 0.657 & 153 & 31 & 32.300 & 1.252 & 18.250 & 2 & $6.8 \mathrm{E}-23$ & + \\
\hline caterpillar39 & 819 & 818 & 33 & 241 & 251.000 & 8.367 & 0.941 & 208 & 34 & 38.500 & 4.275 & 21.364 & 1 & $1.8 \mathrm{E}-20$ & + \\
\hline caterpillar44 & 1034 & 1033 & 37 & 321 & 334.700 & 6.977 & 1.358 & 284 & 39 & 54.000 & 7.732 & 23.906 & 2 & $4.4 \mathrm{E}-16$ & + \\
\hline hypercube11 & 2048 & 11264 & 526 & 1021 & 1022.500 & 0.707 & 600.000 & 495 & 570 & 582.200 & 9.461 & 600.000 & 44 & $1.7 \mathrm{E}-21$ & + \\
\hline hypercube 12 & 4096 & 24576 & 988 & 2046 & 2046.900 & 0.316 & 600.000 & 1058 & 1175 & 1203.300 & 24.208 & 600.000 & 187 & $2.4 \mathrm{E}-18$ & + \\
\hline \multirow[t]{2}{*}{ hypercube 13} & 8192 & 53248 & 1912 & 4095 & 4095.000 & 0.000 & 600.000 & 2183 & 2380 & 2462.400 & 116.956 & 600.000 & 468 & $5.3 \mathrm{E}-05$ & + \\
\hline & Average & & & 191.812 & 195.909 & 2.686 & 22.484 & 131.176 & 88.435 & 93.345 & 4.738 & 29.484 & 27.800 & & \\
\hline
\end{tabular}

Table A.2: Detailed performance comparison of the SAсB and TSCB algorithms over 28 Harwell-Boeing graphs.

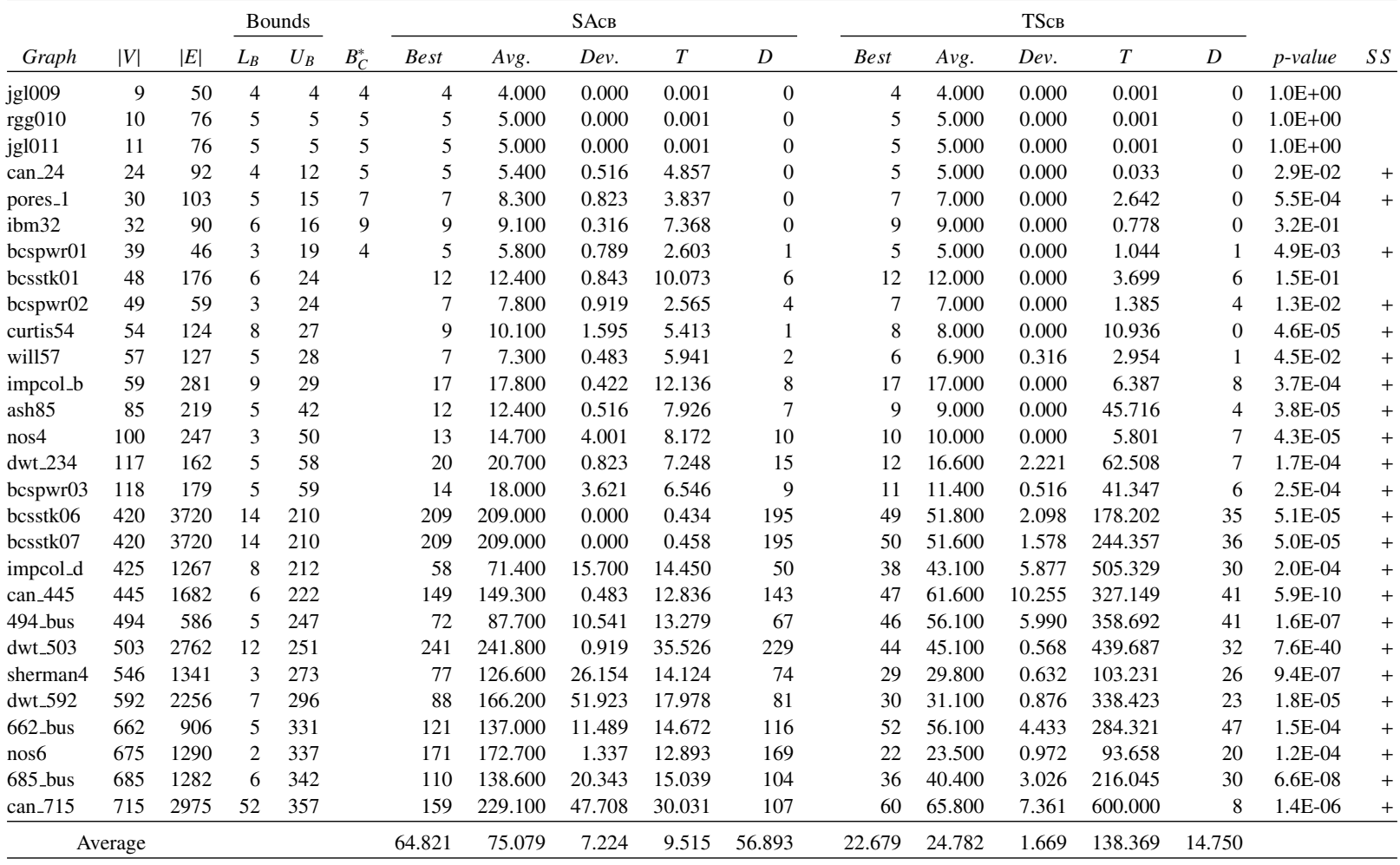

\section{References}

[1] J. Leung, O. Vornberger, J. Witthoff, On some variants of the bandwidth minimization problem, SIAM Journal on Computing 13 (3) (1984) $650-667$.

[2] Y. Lin, The cyclic bandwith problem, Journal of Systems Science and Complexity 7 (3) (1994) 282.

[3] S. N. Bhatt, F. Thomson Leighton, A framework for solving VLSI graph layout problems, Journal of Computer and System Sciences 28 (2) (1984) 300-343.

[4] A. L. Rosenberg, L. Snyder, Bounds on the costs of data encodings, Theory of Computing Systems 12 (1) (1978) 9-39.

[5] F. R. K. Chung, Labelings of graphs, in: L. W. Beineke, R. J. Wilson (Eds.), Selected topics in graph theory volume 3, Academic Press, 1988, Ch. 7, pp. 151-168.

[6] J. Hromkovič, V. Müller, O. Sýkora, I. Vrťo, On embedding interconnection networks into rings of processors, Lecture Notes in Computer Science 605 (1992) 51-62. 
[7] L. H. Harper, Optimal assignment of numbers to vertices, Journal of SIAM 12 (1) (1964) 131-135.

[8] P. C. B. Lam, W. C. Shiu, W. H. Chan, Characterization of graphs with equal bandwidth and cyclic bandwidth, Discrete Mathematics 242 (3) (2002) 283-289.

[9] R. Martí, V. Campos, E. Piñana, A branch and bound algorithm for the matrix bandwidth minimization, European Journal of Operational Research 186 (2) (2008) 513-528.

[10] D. M. Cohen, S. R. Dalal, J. Parelius, G. C. Patton, The combinatorial design approach to automatic test generation, IEEE Software 13 (5) (1996) 83-88.

[11] D. M. Cohen, S. R. Dalal, M. L. Fredman, G. C. Patton, The AETG system: An approach to testing based on combinatorial design, IEEE Transactions on Software Engineering 23 (1997) 437-444.

[12] H. Romero-Monsivais, E. Rodriguez-Tello, G. Ramírez, A new branch and bound algorithm for the cyclic bandwidth problem, Lecture Notes in Artificial Intelligence 7630 (2012) 139-150.

[13] A. Duarte, R. Martí, M. G. C. Resende, R. M. A. Silva, Grasp with path relinking heuristics for the antibandwidth problem, Networks 58 (3) (2011) 171-189.

[14] M. Lozano, A. Duarte, F. Gortázar, R. Martí, Variable neighborhood search with ejection chains for the antibandwidth problem, Journal of Heuristics 18 (6) (2012) 919-938.

[15] Y. Lin, Minimum bandwidth problem for embedding graphs in cycles, Networks 29 (3) (1997) 135-140.

[16] R. Livesley, The analysis of large structural systems, Computer Journal 3 (1) (1960) 34-39.

[17] L. Yixun, Y. Jinjiang, The dual bandwidth problem for graphs, Journal of Zhengzhou University 35 (1) (2003) 1-5.

[18] R. Martí, M. Laguna, F. Glover, V. Campos, Reducing the bandwidth of a sparse matrix with tabu search, European Journal of Operational Research 135 (2) (2001) 211-220.

[19] E. Piñana, I. Plana, V. Campos, R. Martí, GRASP and path relinking for the matrix bandwidth minimization, European Journal of Operational Research 153 (2004) 200-210.

[20] A. Lim, J. Lin, F. Xiao, Particle swarm optimization and hill climbing for the bandwidth minimization problem, Applied Intelligence 26 (3) (2007) 175-182.

[21] E. Rodriguez-Tello, J. K. Hao, J. Torres-Jimenez, An improved simulated annealing algorithm for bandwidth minimization, European Journal of Operational Research 185 (3) (2008) 1319-1335.

[22] N. Mladenovic, D. Urosevic, D. Pérez-Brito, C. G. García-González, Variable neighbourhood search for bandwidth reduction, European Journal of Operational Research 200 (1) (2010) 14-27.

[23] Z. Miller, D. Pritikin, On the separation number of a graph, Networks 19 (6) (1989) 651-666.

[24] W. Yao, Z. Ju, L. Xiaoxu, Dual bandwidth of some special trees, Journal of Zhengzhou University Natural Science Edition 35 (2003) $16-19$.

[25] T. Calamoneri, A. Missini, L. Török, I. Vrt'o, Antibandwidth of complete k-ary trees, Electronic Notes in Discrete Mathematics 24 (2006) 259-266.

[26] L. Török, Antibandwidth of three-dimensional meshes, Electronic Notes in Discrete Mathematics 28 (2007) 161-167. doi:10.1016/j.endm.2007.01.023.

[27] A. Raspaud, H. Schröder, O. Sýkora, L. Török, I. Vrt'o, Antibandwidth and cyclic antibandwidth of meshes and hypercubes, Discrete Mathematics 309 (11) (2009) 3541-3552.

[28] R. Bansal, K. Srivastava, Memetic algorithm for the antibandwidth maximization problem, Journal of Heuristics 17 (1) (2011) $39-60$.

[29] O. Sýkora, L. Török, I. Vrt'o, The cyclic antibandwidth problem, Electronic Notes in Discrete Mathematics 22 (2005) $233-227$.

[30] S. Dobrev, R. Královic, D. Pardubská, L. Török, I. Vrt'o, Antibandwidth and cyclic antibandwidth of hamming graphs, Electronic Notes in Discrete Mathematics 34 (2009) 295-300.

[31] R. Bansal, K. Srivastava, A memetic algorithm for the cyclic antibandwidth maximization problem, Soft Computing 15 (2) (2011) $397-412$.

[32] M. Lozano, A. Duarte, F. Gortázar, R. Martí, A hybrid metaheuristic for the cyclic antibandwidth problem, Knowledge-Based Systems 54 (2013) 103-113.

[33] S. Zhou, Bounding the bandwidths for graphs, Theoretical computer science 249 (2) (2002) 357-368.

[34] E. de Klerk, M. Eisenberg-Nagy, R. Sotirov, On semidefinite programming bounds for graph bandwidth, Technical report, Centrum Wiskunde \& Informatica, (2011).

[35] J. Yuan, S. Zhou, Optimal labelling of unit interval graphs, Applied Mathematics, A Journal of Chinese Universities 10B (3) (1995) 337-344.

[36] F. Glover, M. Laguna, Tabu Search, Kluwer Academic Publishers, 1997.

[37] Z. P. Lü, J. K. Hao, A memetic algorithm for graph coloring, European Journal of Operational Research 203 (1) (2010) 241-250.

[38] Z. P. Lü, J. K. Hao, Adaptive tabu search for course timetabling, European Journal of Operational Research 200 (1) (2010) $235-244$.

[39] P. Galinier, Z. Boujbel, M. Coutinho Fernandes, An efficient memetic algorithm for the graph partitioning problem, Annals of Operations Research 191 (1) (2011) 1-22.

[40] J. Jin, T. G. Crainic, A. Løkketangen, A parallel multi-neighborhood cooperative tabu search for capacitated vehicle routing problems, European Journal of Operational Research 222 (3) (2012) 441-451.

[41] B. Cesaret, C. Oğuz, F. S. Salman, A tabu search algorithm for order acceptance and scheduling, Computers \& Operations Research 39 (6) (2012) 1197-1205.

[42] F. Glover, M. Laguna, Tabu search, in: P. M. Pardalos, D. Z. Du, R. L. Graham (Eds.), Handbook of Combinatorial Optimization, 2nd Edition, Springer, 2013.

[43] R. Kothari, D. Ghosh, Tabu search for the single row facility layout problem using exhaustive 2-opt and insertion neighborhoods, European Journal of Operational Research 224 (1) (2013) 93-100.

[44] Z. P. Lü, J. K. Hao, F. Glover, Neighborhood analysis: a case study on curriculum-based course, Journal of Heuristics 17 (2) (2011) 97-118.

[45] Q. Wu, J. K. Hao, Memetic search for the max-bisection problem, Computers \& Operations Research 40 (1) (2013) 166-179.

[46] J. Chvátalová, Optimal labelling of a product of two paths, Discrete Mathematics 11 (3) (1975) $249-253$.

[47] L. Smithline, Bandwidth of the complete $k$-ary tree, Discrete Mathematics 142 (1-3) (1995) 203-212.

[48] W. A. de Landgraaf, A. E. Eiben, V. Nannen, Parameter calibration using meta-algorithms, in: In proceedings of the IEEE Congress on 
Evolutionary Computation, IEEE Press, 2007, pp. 71-78.

[49] A. Gunawan, H. C. Lau, Lindawati, Fine-tuning algorithm parameters using the design of experiments, Lecture Notes in Computer Science 6683 (2011) 131-145.

[50] L. Gonzalez-Hernandez, J. Torres-Jimenez, MiTS: A new approach of tabu search for constructing mixed covering arrays., Lecture Notes in Artificial Intelligence 6438 (2010) 382-392.

[51] J. Richer, E. Rodriguez-Tello, K. E. Vazquez-Ortiz, Maximum parsimony phylogenetic inference using simulated annealing, in: O. Schütze, C. Coello Coello, A. Tantar, E. Tantar, P. Bouvry, P. Del Moral, P. Legrand (Eds.), EVOLVE - A Bridge between Probability, Set Oriented Numerics, and Evolutionary Computation II, Vol. 175 of Advances in Intelligent Systems and Computing, Springer, 2013, pp. 189-203.

[52] C. J. Colbourn, Combinatorial aspects of covering arrays, Le Matematiche 58 (2004) 121-167.

[53] E. Rodriguez-Tello, J. Torres-Jimenez, Memetic algorithms for constructing binary covering arrays of strength three, Lecture Notes in Computer Science 5975 (2010) 86-97.

[54] H. Toutenburg, Shalabh, Statistical Analysis of Designed Experiments, 3rd Edition, Sp, 2009.

[55] T. Tsuchiya, Y. Takenaka, H. Taguchi, Multidisciplinary design optimization for hypersonic experimental vehicle, AIAA Journal 45 (7) (2007) 1655-1662.

[56] A. Azadeh, Z. S. Faiz, A meta-heuristic framework for forecasting household electricity consumption, Applied Soft Computing 11 (1) (2011) $614-620$.

[57] P. Samui, Slope stability analysis using multivariate adaptive regression spline, in: X. S. Yang, A. H. Gandomi, S. Talatahari, A. H. Alavi (Eds.), Metaheuristics in Water, Geotechnical and Transport Engineering, Elsevier, 2013, Ch. 14, pp. 327-342.

[58] H. G. Santos, L. S. Ochi, M. J. F. Souza, A tabu search heuristic with efficient diversification strategies for the class/teacher timetabling problem, ACM Journal of Experimental Algorithmics 10 (Article No. 2.9) (2005) 1-16.

[59] T. James, C. Rego, F. Glover, Multistart tabu search and diversification strategies for the quadratic assignment problem, IEEE Transactions on Systems, Man, and Cybernetics, Part A 39 (3) (2009) 579-596.

[60] P. F. Stadler, Correlation in landscapes of combinatorial optimization problems, Europhysics Letters 20 (1992) $479-482$.

[61] E. Pitzer, M. Affenzeller, A comprehensive survey on fitness landscape analysis, in: J. Fodor, R. Klempous, C. P. Suárez-Araujo (Eds.), Recent Advances in Intelligent Engineering Systems, Vol. 378 of Studies in Computational Intelligence, Springer, 2012, Ch. 8, pp. 161-191.

[62] E. Talbi, Metaheuristics: From design to implementation, John Wiley \& Sons, 2009. 